\title{
QUASI-PERIODIC SOLUTIONS OF THE RELATIVISTIC TODA HIERARCHY
}

\author{
DONG GONG* and XIANGUO GENG ${ }^{\dagger}$ \\ Department of Mathematics, Zhengzhou University \\ 100 Kexue Road, Zhengzhou \\ Henan 450001, P. R. China \\ *gongdong2004@126.com \\ ${ }^{\dagger} x g g e n g @ z z u . e d u . c n$
}

Received 7 May 2012

Accepted 18 August 2012

Published 31 December 2012

\begin{abstract}
On the basis of the theory of algebraic curves, the continuous flow and discrete flow related to the relativistic Toda hierarchy are straightened out using the Abel-Jacobi coordinates. The meromorphic function and the Baker-Akhiezer function are introduced on the hyperelliptic curve. Quasiperiodic solutions of the relativistic Toda hierarchy are constructed with the help of the asymptotic properties and the algebro-geometric characters of the meromorphic function and the hyperelliptic curve.
\end{abstract}

Keywords: Relativistic Toda hierarchy; quasi-periodic solutions.

\section{Introduction}

The relativistic Toda lattice

$$
\begin{aligned}
& a_{t}(n)=\frac{1}{2} a(n)\left[a^{2}(n+1)-a^{2}(n-1)+b(n+1)-b(n)\right], \\
& b_{t}(n)=b(n)\left[a^{2}(n)-a^{2}(n-1)\right] .
\end{aligned}
$$

was first introduced by Ruijsenaars [16] and has been extensively studied by many authors. For instance, its integrability has been proved by the author himself, and in a list of papers by Bruschi and Ragnisco, in the case of a finite lattice (periodic case and free ends) [2-4]. Oevel, Fuchssteiner and Zhang investigated the integrability structure of the infinite relativistic Toda lattice from the Lie-algebraic point of view [14]. Alber found a nonlinear family of relativistic Toda lattices with corresponding stationary and dynamical systems, and reduced the finite-gap problem to the Jacobi inversion problem [1]. Ohta, Kajiwara, Matusukidara and Satsuma obtained its Casorati determinant solution [15]. Suris studied algebraic structure of discrete-time and relativistic Toda lattices [17]. Under a constraint between potentials and eigenfunctions, the discrete spectral problem associated with the 


\section{Gong $\& 3$ X. Geng}

relativistic Toda lattice is nonlinearized so as to be an integrable symplectic map of Neumann type, and the calculation of the finite-band solutions of the relativistic Toda lattice is reduced to the solution of a system of ordinary differential equations plus a simple iterative process of the symplectic map [18]. The relation between 2+1-dimensional modified Toda lattice and the relativistic Toda flows are revealed in [7]. A Darboux transformation for the relativistic Toda lattice is constructed, by which exact solutions of the relativistic Toda lattice equation are presented [19].

We recall here the basic definitions for the relativistic Toda lattice and its various different forms. In terms of the canonically conjugate variables $(q(n, t), p(n, t))$, the infinite relativistic Toda system is defined by the following Hamiltonian $[2,14,16]$

$$
\begin{aligned}
H(q, p)= & \sum_{n \in \mathbb{Z}}\left\{\exp (p(n))[1+\exp (q(n-1)-q(n))]^{\frac{1}{2}}\right. \\
& \left.\times[1+\exp (q(n)-q(n+1))]^{\frac{1}{2}}-2\right\}
\end{aligned}
$$

whose canonical equations of motion of the Hamiltonian system reads

$$
\begin{aligned}
& q_{t}(n)=c(n), \\
& p_{t}(n)=\frac{1}{2} d(n-1)(c(n)+c(n-1))-\frac{1}{2} d(n)(c(n)+c(n+1)),
\end{aligned}
$$

where

$$
\begin{aligned}
& c(n)=\exp (p(n))[1+\exp (q(n-1)-q(n-1))]^{\frac{1}{2}}[1+\exp (q(n)-q(n+1))]^{\frac{1}{2}}, \\
& d(n)=\frac{\exp (q(n)-q(n+1))}{1+\exp (q(n)-q(n+1))} .
\end{aligned}
$$

Substituting (1.4) into (1.3) and canceling variable $p(n)$, the equations of motion (1.3) are written in Newtonian form

$$
q_{t t}(n)=q_{t}(n)\left(q_{t}(n-1) \frac{\exp (q(n-1)-q(n))}{1+\exp (q(n-1)-q(n))}-q_{t}(n+1) \frac{\exp (q(n)-q(n+1))}{1+\exp (q(n)-q(n+1))}\right) .
$$

Differentiating (1.4) with respect to $t$ and substituting (1.3) yield the following lattice [3]

$$
\begin{aligned}
& c_{t}(n)=c(n-1) c(n) d(n-1)-c(n) c(n+1) d(n), \\
& d_{t}(n)=d(n)(1-d(n))(c(n)-c(n+1)) .
\end{aligned}
$$

A further useful form of the relativistic Toda lattice can be obtained from (1.6) by setting

$$
r(n)=c(n) d(n), \quad s(n)=c(n)(1-d(n)),
$$

so that we have [4]

$$
\begin{aligned}
& s_{t}(n)=s(n)(r(n-1)-r(n)), \\
& r_{t}(n)=r(n)(s(n)-s(n+1)+r(n-1)-r(n+1)),
\end{aligned}
$$


which can be written as (1.1) in terms of new variables

$$
r(n)=-a^{2}(n), \quad s(n)=-b(n) .
$$

The main aim of this paper is to construct quasi-periodic solutions of the relativistic Toda hierarchy based on the approaches in [5, 6, 8-10]. This is realized through three main steps. First, based on the resulting Lax representation, the relativistic Toda hierarchy is decomposed into the solvable ordinary differential equations. Second, the determinant of Lax matrix serves as a base to construct the associated algebraic curve and the AbelJacobi coordinates, through which the continuous flows and discrete ones determined by the time variable $t_{\underline{m}}$ and discrete variable $n$, respectively, are straightened out and the linear superposition yields the solutions of the relativistic Toda hierarchy, expressed in the Abel-Jacobi coordinates. Here straightening out of the various flows means that the AbelJacobi coordinate is a linear function of the associated flow variables $t_{\underline{m}}$ and $n$. Third, an inverse procedure is indispensable in transforming the explicit solution in the original coordinates. The main tool in our theory is the asymptotic properties and the algebrogeometric characters of the meromorphic function and the hyperelliptic curve.

The outline of this paper is as follows. In Sec. 2, we introduce the Lenard gradients and derive the relativistic Toda hierarchy with the aid of the stationary zero-curvature equation, in which the first nontrivial member is the relativistic Toda lattice. In Sec. 3, we introduce a Lax matrix and establish a direct relation between the elliptic variables and the potentials. The relativistic Toda hierarchy is separated into solvable ordinary differential equations. In Sec. 4, the hyperelliptic Riemann surface of arithmetic genus $N$ and the Abel-Jacobi coordinates are introduced from which the corresponding various flows are straightened out, including the continuous and discrete ones. In Sec. 5, quasi-periodic solutions of the relativistic Toda hierarchy are constructed in terms of the Riemann theta functions according to the asymptotic properties and the algebro-geometric characters of the meromorphic function on the hyperelliptic curve.

\section{The Relativistic Toda Hierarchy}

In this section, we construct the relativistic Toda hierarchy. Throughout this paper we suppose the following hypothesis.

Hypothesis 2.1. Assume that $a$ and $b$ satisfy

$$
\begin{aligned}
& a(\cdot, t), b(\cdot, t) \in \mathbb{C}^{\mathbb{Z}}, \quad t \in \mathbb{R}, \quad a(n, \cdot), b(n, \cdot) \in C^{1}(\mathbb{R}), \quad n \in \mathbb{Z}, \\
& a(n, t) b(n, t) \neq 0, \quad(n, t) \in \mathbb{Z} \times \mathbb{R},
\end{aligned}
$$

where $\mathbb{C}^{\mathbb{Z}}$ denotes the set of all complex-valued sequences indexed by $\mathbb{Z}$. For the sake of convenience, we denote by $E^{ \pm}$the shift operators acting on complex-valued sequences $f=\{f(n)\}_{n \in \mathbb{Z}}$ according to

$$
\left(E^{ \pm} f\right)(n)=f(n \pm 1), \quad n \in \mathbb{Z}
$$

and define difference operators by $\triangle=E-1, \triangle^{*}=E^{-}-1$. Moreover, we will frequently use the notation

$$
f^{ \pm}=E^{ \pm} f, \quad f \in \mathbb{C}^{\mathbb{Z}} .
$$


D. Gong $\& 3$ X. Geng

Let us consider the discrete spectral problem

$$
\chi_{n+1}=U_{n} \chi_{n}, \quad U_{n}=\frac{1}{a(n)}\left(\begin{array}{cc}
0 & -a^{2}(n) \\
1 & \lambda-\lambda^{-1} b(n)
\end{array}\right),
$$

where $a(n)$ and $b(n)$ are two potentials, and $\lambda$ is a constant spectral parameter. To derive the relativistic Toda hierarchy, we introduce the Lenard gradient sequences

$$
\begin{array}{ll}
K_{n} S_{j-1}(n)=J_{n} S_{j}(n), & J_{n} S_{-1}(n)=0, \quad j \geq 0 \\
K_{n} \hat{S}_{j-1}(n)=J_{n} \hat{S}_{j}(n), & K_{n} \hat{S}_{0}(n)=0, \quad j \leq 0
\end{array}
$$

where $S_{j}(n)=\left(S_{j}^{(1)}(n), S_{j}^{(2)}(n)\right)^{T}, \hat{S}_{j}(n)=\left(\hat{S}_{j}^{(1)}(n), \hat{S}_{j}^{(2)}(n)\right)^{T}$, and take

$$
S_{-1}(n)=\left(\begin{array}{c}
2 a(n) \\
1
\end{array}\right), \quad \hat{S}_{0}(n)=\left(\begin{array}{c}
0 \\
\frac{1}{b(n)}
\end{array}\right)
$$

as a starting point of (2.2) or (2.3), and two skew-symmetric matrix operators are defined as $K_{n}=\left(\begin{array}{cc}\frac{1}{2} a(n)\left(\triangle-\triangle^{*}\right) a(n) & a(n) \triangle b(n) \\ -b(n) \triangle^{*} a(n) & 0\end{array}\right), \quad J_{n}=\left(\begin{array}{cc}0 & a(n) \triangle \\ -\triangle^{*} a(n) & 2\left(\triangle^{*} a^{2}(n)-a^{2}(n) \triangle\right)\end{array}\right)$.

It is easy to see that

$$
\begin{aligned}
\operatorname{ker} J_{n} & =\left\{c_{1} S_{-1}(n)+c_{2} S_{-2}(n) \mid \forall c_{1}, c_{2} \in \mathbb{R}\right\}, \\
\operatorname{ker} K_{n} & =\left\{c_{1} \hat{S}_{0}(n)+c_{2} \hat{S}_{1}(n) \mid \forall c_{1}, c_{2} \in \mathbb{R}\right\},
\end{aligned}
$$

where $c_{1}$ and $c_{2}$ are two arbitrary constants, $S_{-2}=\hat{S}_{1}=\left(\frac{1}{a(n)}, 0\right)^{T}$. Then $S_{j}(j \geq 0)$ and $\hat{S}_{j}(j \leq 0)$ are uniquely determined by $(2.2)$ or $(2.3)$, respectively, up to a term $c_{1} S_{-1}(n)+$ $c_{2} S_{-2}(n)$ or $c_{1} \hat{S}_{0}(n)+c_{2} \hat{S}_{1}(n)$, which are always assumed to be zero. For example, the first two members are

$$
\begin{gathered}
S_{0}(n)=\left(\begin{array}{c}
2 a(n)\left(a^{2}(n+1)+a^{2}(n)+a^{2}(n-1)+b(n+1)+b(n)\right) \\
a^{2}(n)+a^{2}(n-1)+b(n)
\end{array}\right), \\
\hat{S}_{-1}(n)=\left(\begin{array}{c}
-\frac{2 a(n)}{b(n+1) b(n)} \\
\frac{1}{b^{2}(n)}\left(1+\frac{a^{2}(n)}{b(n+1)}+\frac{a^{2}(n-1)}{b(n-1)}\right)
\end{array}\right) .
\end{gathered}
$$

Assume that the time evolution of the eigenfunction $\chi_{n}$ obeys the differential equation

$$
\chi_{n, t_{\underline{m}}}=V_{n}^{(\underline{m})} \chi_{n}, \quad V_{n}^{(\underline{m})}=\left(\begin{array}{cc}
A^{(\underline{m})}(n) & \lambda B^{(\underline{m})}(n) \\
\lambda C^{(\underline{m})}(n) & -A^{(\underline{m})}(n)
\end{array}\right), \quad \underline{m}=\left(m_{1}, m_{2}\right) \in \mathbb{N}_{0}^{2},
$$


where $A^{(\underline{m})}(n), B^{(\underline{m})}(n), C^{(\underline{m})}(n)$ are polynomials of the spectral parameter $\lambda$, which take the form

$$
\begin{aligned}
& A^{(\underline{m})}(n)=b(n) S^{(2)}(n)-\frac{1}{2} \triangle^{*} a_{n} S^{(1)}(n)-\lambda^{2} S^{(2)}(n), \\
& B^{(\underline{m})}(n)=-2 a^{2}(n-1) S^{(2)}(n), \quad C^{(\underline{m})}(n)=2 S^{(2)}(n), \\
& S^{(1)}(n)=\alpha \sum_{j=0}^{m_{1}} S_{j-1}^{(2)}(n) \lambda^{2 m_{1}-2 j}+\beta \sum_{j=0}^{-m_{2}+1} \hat{S}_{j}^{(1)}(n) \lambda^{-2 m_{2}-2 j}, \\
& S^{(2)}(n)=\alpha \sum_{j=0}^{m_{1}} S_{j-1}^{(2)}(n) \lambda^{2 m_{1}-2 j}+\beta \sum_{j=0}^{-m_{2}+1} \hat{S}_{j}^{(2)}(n) \lambda^{-2 m_{2}-2 j} .
\end{aligned}
$$

Then the compatibility condition $(\partial / \partial t) E \chi_{n}=E(\partial / \partial t) \chi_{n}$ of (2.1) and (2.7) yields the zero-curvature equation, $U_{n, t_{\underline{m}}}+U_{n} V_{n}^{(\underline{m})}-V_{n+1}^{(\underline{m})} U_{n}=0$, which is equivalent to a hierarchy of the relativistic Toda lattices:

$$
(a(n), b(n))_{t_{\underline{m}}}^{T}=\alpha X_{m_{1}}(n)-\beta X_{-m_{2}}(n), \quad m_{1} \geq 0, m_{2} \geq 1,
$$

where $X_{m_{1}}=K_{n} S_{m_{1}-1}=J_{n} S_{m_{1}}, X_{-m_{2}}=K_{n} \hat{S}_{-m_{2}}=J_{n} \hat{S}_{-m_{2}+1}$. The first member in (2.9) is the relativistic Toda lattice

$$
\begin{aligned}
a_{t_{(0,1)}}(n)= & \alpha a(n)\left(a^{2}(n+1)-a^{2}(n-1)\right. \\
& +b(n+1)-b(n))-\beta a(n)\left(\frac{1}{b(n+1)}-\frac{1}{b(n)}\right), \\
b_{t_{(0,1)}}(n)= & 2 \alpha b(n)\left(a^{2}(n)-a^{2}(n-1)\right)-2 \beta\left(\frac{a^{2}(n-1)}{b(n-1)}-\frac{a^{2}(n)}{b(n+1)}\right) .
\end{aligned}
$$

Equation (2.10) is, respectively, reduced to the two members in the relativistic Toda lattice hierarchy

$$
\begin{aligned}
& a_{t}(n)=\frac{1}{2} a(n)\left(a^{2}(n+1)-a^{2}(n-1)+b(n+1)-b(n)\right), \\
& b_{t}(n)=b(n)\left(a^{2}(n)-a^{2}(n-1)\right),
\end{aligned}
$$

for $\alpha=\frac{1}{2}, \beta=0, t=t_{(0,1)}$, and

$$
\begin{aligned}
& a_{x}(n)=\frac{1}{2} a(n)\left(\frac{1}{b(n+1)}-\frac{1}{b(n)}\right), \\
& b_{x}(n)=\frac{a^{2}(n-1)}{b(n-1)}-\frac{a^{2}(n)}{b(n+1)},
\end{aligned}
$$

for $\alpha=0, \beta=-\frac{1}{2}, x=t_{(0,1)}$.

\section{Evolution of the Elliptic Variables}

In what follows, we will establish a relation between the elliptic variables $\mu_{j}(n)$ (see $(3.18)$ and [12]) and the potentials $a(n), b(n)$ in the discrete spectral problem $(2.1)$. Let $\psi(n)=$ $\left(\psi_{1}(n), \psi_{2}(n)\right)^{T}$ and $\varphi(n)=\left(\varphi_{1}(n), \varphi_{2}(n)\right)^{T}$ be two basic solutions of $(2.1)$ and $(2.7)$. We 
introduce a Lax matrix

$$
W_{n}=\frac{1}{2}\left(\psi(n) \varphi(n)^{T}+\varphi(n) \psi(n)^{T}\right)\left(\begin{array}{cc}
0 & -1 \\
1 & 0
\end{array}\right)=\left(\begin{array}{cc}
G(n) & \lambda F(n) \\
\lambda H(n) & -G(n)
\end{array}\right)
$$

which satisfies the Lax equations

$$
W_{n+1} U_{n}-U_{n} W_{n}=0, \quad W_{n, t_{\underline{m}}}=\left[V_{n}^{(\underline{m})}, W_{n}\right]
$$

Therefore, det $W$ is a constant independent of $n$ and $t_{\underline{m}}$. In fact, we obtain by the first expression of (3.2) that $W_{n+1}=U_{n} W_{n} U_{n}^{-1}$. Then $\operatorname{det} \bar{W}_{n+1}=\operatorname{det} W_{n}$, which means that det $W_{n}$ is independent of $n$. In a way similar to the continuous case, a direct calculation shows that $(\operatorname{det} W)_{t_{\underline{m}}}=0$.

In the following, we shall solve the Lax equations (3.2), by which we derive explicit expressions of $G(n), F(n), H(n)$ in the Lax matrix $W_{n}$ and show them to be polynomials of $\lambda$ and $\lambda^{-1}$. Equation (3.2) can be written as

$$
\begin{aligned}
& F(n+1)+a^{2}(n) H(n)=0 \\
& \left(\lambda^{2}-b(n)\right) F(n+1)-a^{2}(n)(G(n+1)+G(n))=0, \\
& \left(\lambda^{2}-b(n)\right) H(n)+(G(n+1)+G(n))=0 \\
& \left(\lambda^{2}-b(n)\right) \triangle G(n)+\lambda^{2} a^{2}(n) H(n+1)+\lambda^{2} F(n)=0,
\end{aligned}
$$

and

$$
\begin{aligned}
& G(n)_{t_{\underline{m}}}=\lambda^{2} B^{(\underline{m})} H(n)-\lambda^{2} C^{(\underline{m})}(n) F(n), \\
& F(n)_{t_{\underline{m}}}=2 A^{(\underline{m})}(n) F(n)-2 B^{(\underline{m})}(n) G(n), \\
& H(n)_{t_{\underline{m}}}=2 C^{(\underline{m})}(n) G(n)-2 A^{(\underline{m})}(n) H(n) .
\end{aligned}
$$

Suppose functions $G(n), F(n)$ and $H(n)$ are finite-order polynomials in $\lambda$ :

$$
\begin{aligned}
& G(n)=b_{n} Q^{(2)}(n)-\frac{1}{2} \triangle^{*} a(n) Q^{(1)}(n)-\lambda^{2} Q^{(2)}(n), \\
& F(n)=-2 a^{2}(n-1) Q^{(2)}(n-1), \quad H(n)=2 Q^{(2)}(n), \\
& Q^{(1)}(n)=\sum_{j=0}^{N_{1}} Q_{j-1}^{(1)}(n) \lambda^{2 N_{1}-2 j}+\sum_{j=0}^{-N_{2}+1} \hat{Q}_{j}^{(1)}(n) \lambda^{-2 N_{2}-2 j} \\
& Q^{(2)}(n)=\sum_{j=0}^{N_{1}} Q_{j-1}^{(2)}(n) \lambda^{2 N_{1}-2 j}+\sum_{j=0}^{-N_{2}+1} \hat{Q}_{j}^{(2)}(n) \lambda^{-2 N_{2}-2 j}
\end{aligned}
$$

Substituting (3.5) into (3.3) yields

$$
\begin{aligned}
& K_{n} Q_{j-1}(n)=J_{n} Q_{j}(n), \quad J_{n} Q_{-1}(n)=0, \quad j \geq 0 \\
& K_{n} \hat{Q}_{j-1}(n)=J_{n} \hat{Q}_{j}(n), \quad K_{n} \hat{Q}_{0}(n)=0, \quad j \leq 0, \\
& K_{n} Q_{N_{1}-1}(n)=J_{n} \hat{Q}_{-N_{2}+1}(n),
\end{aligned}
$$


with $Q_{j}(n)=\left(Q_{j}^{(1)}(n), Q_{j}^{(2)}(n)\right)^{T},(j \geq 0) ; \hat{Q}_{j}(n)=\left(\hat{Q}_{j}^{(1)}(n), \hat{Q}_{j}^{(2)}(n)\right)^{T},(j \leq 0)$. It is easy to see that the equation $J_{n} Q_{-1}=0$ and $K_{n} \hat{Q}_{0}(n)=0$ have the general solution:

$$
\begin{gathered}
Q_{-1}(n)=\alpha_{0,+} S_{-1}(n)+\hat{\alpha}_{0,+} S_{-2}(n), \\
\hat{Q}_{0}(n)=\alpha_{0,-} \hat{S}_{0}(n)+\hat{\alpha}_{0,-} \hat{S}_{1}(n),
\end{gathered}
$$

where $\alpha_{0,+}, \hat{\alpha}_{0,+}$ and $\alpha_{0,-}, \hat{\alpha}_{0,-}$ are constants. Noticing $S_{-2}=\hat{S}_{1} \in$ ker $K_{n} \cap$ ker $J_{n}$, acting with the operators $\left(J_{n}^{-1} K_{n}\right)^{k+1}$ and $\left(K_{n}^{-1} J_{n}\right)^{k}$, respectively, on (3.7) and (3.8). We obtain from (3.6) and (2.2) that

$$
\begin{aligned}
Q_{k}(n) & =\sum_{j=0}^{k} \alpha_{j,+} S_{k-j}(n)+\alpha_{k+1,+} S_{-1}(n)+\hat{\alpha}_{k+1,+} S_{-2}(n), \quad 0 \leq k \leq N_{1}, \\
\hat{Q}_{-k}(n) & =\sum_{j=0}^{k} \alpha_{j,-} \hat{S}_{-k+j}(n)+\hat{\alpha}_{k,-} S_{-2}(n), \quad 0 \leq k \leq N_{2},
\end{aligned}
$$

where $\alpha_{1,+}, \ldots, \alpha_{k+1,+}, \hat{\alpha}_{1,+}, \ldots, \hat{\alpha}_{k+1,+}, \alpha_{1,-}, \ldots, \alpha_{k,-} \hat{\alpha}_{1,-}, \ldots, \hat{\alpha}_{k,-}$ are constants. Substituting (3.9) and (3.10) into the final equation in (3.6) gives rise to the $N$-order stationary relativistic Toda lattice

$$
\alpha_{0,+} X_{N_{1}}(n)+\cdots+\alpha_{N_{1},+} X_{0}(n)+\alpha_{N_{2}-1,-} X_{-1}(n)+\cdots+\alpha_{0,-} X_{-N_{2}}(n)=0 .
$$

From (3.5), (3.7)-(3.10), we have

$$
\begin{aligned}
& G(n)=\sum_{j=0}^{N_{1}+1} g_{j,+}(n) \lambda^{2\left(N_{1}+1\right)-2 j}+\sum_{j=0}^{N_{2}-1} g_{j,-}(n) \lambda^{-2 N_{2}+2 j}, \\
& F(n)=\sum_{j=0}^{N_{1}} f_{j,+}(n) \lambda^{2 N_{1}-2 j}+\sum_{j=0}^{N_{2}-1} f_{j,-}(n) \lambda^{-2 N_{2}+2 j}, \\
& H(n)=\sum_{j=0}^{N_{1}} h_{j,+}(n) \lambda^{2 N_{1}-2 j}+\sum_{j=0}^{N_{2}-1} h_{j,-}(n) \lambda^{-2 N_{2}+2 j},
\end{aligned}
$$

where

$$
\begin{aligned}
& g_{0,+}(n)=-\alpha_{0,+}, \quad g_{1,+}(n)=-2 \alpha_{0,+} a^{2}(n-1)-\alpha_{1,+}, \\
& f_{0,+}(n)=-2 \alpha_{0,+} a^{2}(n-1) \\
& f_{1,+}(n)=-2 \alpha_{0,+} a^{2}(n-1)\left(a^{2}(n-1)+a^{2}(n-2)+b(n-1)\right)-2 \alpha_{1,+} a^{2}(n-1), \\
& h_{0,+}(n)=2 \alpha_{0,+}, \quad h_{1,+}(n)=2 \alpha_{0,+}\left(a^{2}(n)+a^{2}(n-1)+b(n)\right)+2 \alpha_{1,+}, \\
& g_{0,-}(n)=\alpha_{0,-}, \quad g_{1,-}(n)=\frac{2 \alpha_{0,-} a^{2}(n-1)}{b(n) b(n-1)}+\alpha_{1,-}, \\
& f_{0,-}(n)=-\frac{2 \alpha_{0,-} a^{2}(n-1)}{b(n-1)}
\end{aligned}
$$




$$
\begin{aligned}
& f_{1,-}(n)=-\frac{2 \alpha_{0,-} a^{2}(n-1)}{b^{2}(n-1)}\left(1+\frac{a^{2}(n-1)}{b(n)}+\frac{a^{2}(n-2)}{b(n-2)}\right)-\frac{2 \alpha_{1,-} a^{2}(n-1)}{b(n-1)} \\
& h_{0,-}(n)=\frac{2 \alpha_{0,-}}{b(n)}, \quad h_{1,-}(n)=\frac{2 \alpha_{0,-}}{b^{2}(n)}\left(1+\frac{a^{2}(n)}{b(n+1)}+\frac{a^{2}(n-1)}{b(n-1)}\right)+\frac{2 \alpha_{1,-}}{b(n)} .
\end{aligned}
$$

Subsequently, it will be useful to work with the homogeneous coefficients, $\hat{g}_{j, \pm}, \hat{f}_{j, \pm}$, and $\hat{h}_{j, \pm}$, defined by the vanishing of all summation constants $\alpha_{k, \pm}$ for $k=1,2, \ldots, j$, and $\alpha_{0, \pm}=1$,

$$
\begin{aligned}
& \hat{g}_{0,+}(n)=-1, \quad \hat{g}_{0,-}(n)=1, \quad \hat{g}_{j, \pm}(n)=\left.g_{j, \pm}(n)\right|_{\alpha_{0, \pm}=1, \alpha_{k, \pm}=0, k=1, \ldots, j}, \\
& \hat{f}_{0,+}(n)=-2 a^{2}(n-1), \quad \hat{f}_{0,-}(n)=-\frac{2 a^{2}(n-1)}{b(n-1)}, \\
& \hat{f}_{j, \pm}(n)=\left.f_{j, \pm}(n)\right|_{\alpha_{0, \pm}=1, \alpha_{k, \pm}=0, k=1, \ldots, j}, \\
& \hat{h}_{0,+}(n)=2, \quad \hat{h}_{0,-}(n)=\frac{2}{b(n)}, \quad \hat{h}_{j, \pm}(n)=\left.h_{j, \pm}(n)\right|_{\alpha_{0, \pm}=1, \alpha_{k, \pm}=0, k=1, \ldots, j}, \quad j \in \mathbb{N} .
\end{aligned}
$$

By induction one infers that

$$
\begin{gathered}
g_{j, \pm}(n)=\sum_{l=0}^{k} \alpha_{j-l, \pm} \hat{g}_{k, \pm}(n), \quad f_{j, \pm}(n)=\sum_{l=0}^{k} \alpha_{j-l, \pm} \hat{f}_{k, \pm}(n), \\
h_{j, \pm}(n)=\sum_{l=0}^{k} \alpha_{j-l, \pm} \hat{h}_{k, \pm}(n) .
\end{gathered}
$$

Remark 3.1. As an useful tool to distinguish between nonhomogeneous and homogeneous quantities $g_{j, \pm}, f_{j, \pm}, h_{j, \pm}$, and $\hat{g}_{j, \pm}, \hat{f}_{j, \pm}, \hat{h}_{j, \pm}$, respectively, we now introduce the notion of degree as follows. Denote

$$
f=f(n)_{n \in Z} \in \mathbb{C}^{\mathbb{Z}}, \quad f^{(r)}=S^{(r)} f, \quad S^{(r)}=\left\{\begin{array}{ll}
\left(S^{+}\right)^{r}, & r \geq 0, \\
\left(S^{-}\right)^{-r}, & r<0 .
\end{array} \quad r \in \mathbb{Z},\right.
$$

and defines $\operatorname{deg}\left(a^{(r)}\right)=1, \operatorname{deg}\left(b^{(r)}\right)=2, r \in \mathbb{Z}$.

Using (3.5), (3.6) and (3.11), then results in

$$
\begin{array}{rll}
\operatorname{deg}\left(\hat{g}_{l,+}\right)=2 l, & \operatorname{deg}\left(\hat{g}_{l,-}\right)=-2 l, & l \in \mathbb{N}_{0}, \\
\operatorname{deg}\left(\hat{f}_{l,+}\right)=2 l+2, & \operatorname{deg}\left(\hat{f}_{l,-}\right)=-2 l, & l \in \mathbb{N}_{0}, \\
\operatorname{deg}\left(\hat{h}_{l,+}\right)=2 l, & \operatorname{deg}\left(\hat{h}_{l,-}\right)=-2 l-2, & l \in \mathbb{N}_{0} .
\end{array}
$$

Noticing (3.5) and (3.11), we can write $F(n)$ and $H(n)$ as finite products which take the form

$$
\begin{aligned}
& F(n)=-2 \alpha_{0,+} a^{2}(n-1) z^{-N_{2}} \prod_{j=1}^{N}\left(z-\mu_{j}(n-1)\right), \\
& H(n)=2 \alpha_{0,+} z^{-N_{2}} \prod_{j=1}^{N}\left(z-\mu_{j}(n)\right), \quad N=N_{1}+N_{2},
\end{aligned}
$$


where $z=\lambda^{2}$, and the roots $\left\{\mu_{j}(n)\right\},\left\{\mu_{j}^{-}(n)\right\}$ are called elliptic variables. Comparing the coefficients of $z$ in the expressions of $F(n), H(n)$ in (3.11) and (3.18) readily yields trace formulas for $f_{l, \pm}, h_{l, \pm}$ in terms of symmetric functions of $\mu_{j}(n)$ and $\mu_{j}^{-}(n)$, respectively. For simplicity, we just record the simplest cases

$$
\begin{gathered}
a^{2}(n)+a^{2}(n-1)+b(n)=-\sum_{j=1}^{N} \mu_{j}(n)-\frac{\alpha_{1,+}}{\alpha_{0,+}}, \\
\frac{1}{b(n)}=\frac{\alpha_{0,+}}{\alpha_{0,-}}(-1)^{N} \prod_{j=1}^{N} \mu_{j}(n) .
\end{gathered}
$$

Since det $W_{n}$ is a polynomial that only depends on $z$, whose coefficients are constants of the $n$-flow and $t_{\underline{m}}$-flow, we have

$$
-\operatorname{det} W_{n}=G^{2}(n)+z F(n) H(n)=R(z),
$$

from which we obtain

$$
\left.G(n)\right|_{z=\mu_{k}(n)}=\sqrt{R\left(\mu_{k}(n)\right)}
$$

Noticing (3.4) and (3.18), we get

$$
\begin{aligned}
\left.H(n)_{t_{\underline{m}}}\right|_{z=\mu_{k}(n)} & =-2 \alpha_{0,+} \mu_{k, t_{\underline{m}}}(n) \mu_{k}(n)^{-N_{2}} \prod_{\substack{j=1 \\
j \neq k}}^{N}\left(\mu_{k}(n)-\mu_{j}(n)\right) \\
& =\left.2 \sqrt{R\left(\mu_{k}(n)\right)} C^{(\underline{m})}(n)\right|_{z=\mu_{k}(n)},
\end{aligned}
$$

which means the Dubrovin equations for the time variation of $\mu_{j}(n)$

$$
\mu_{k, \underline{t_{\underline{m}}}}(n)=-\frac{\left.\sqrt{R\left(\mu_{k}(n)\right)} C^{(\underline{m})}(n)\right|_{z=\mu_{k}(n)} \mu_{k}(n)^{N_{2}}}{\alpha_{0,+}+\prod_{\substack{j=1 \\ j \neq k}}^{N}\left(\mu_{k}(n)-\mu_{j}(n)\right)},
$$

with $1 \leq k \leq N, \underline{m}=\left(m_{1}, m_{2}\right), m_{1} \geq 0, m_{2} \geq 1$, and

$$
\left.C^{(\underline{m})}(n)\right|_{z=\mu_{k}}=2 \alpha \sum_{j=0}^{m_{1}} S_{j-1}^{(2)}(n) \mu_{k}^{m_{1}-j}+2 \beta \sum_{j=0}^{-m_{2}+1} \hat{S}_{j}^{(2)}(n) \mu_{k}^{-m_{2}-j} .
$$

\section{Straightening Out of Various Flows}

In the section, we first define a hyperelliptic curve $\mathcal{K}_{N}$ with the aid of the determinant for Lax matrix $W_{n}$. Then we introduce a fundamental meromorphic function and Abel-Jacobi coordinates on $\mathcal{K}_{N}$. The corresponding continuous flow and discrete flow are straightened out under the Abel--Jacobi coordinates. Here the meaning of straightening out of the various flows is that the velocities of the Abel-Jacobi coordinate along the discrete $n$-flow and continuous $t_{\underline{m}}$-flow are constant (see Theorems 4.6 and 4.9 ).

According to (3.1) and (3.2), one infers that the expression, $R(z)=G^{2}+z F H=$ - det $W_{n}$, is a constant independent of $n$ and $t_{\underline{m}}$ with $z=\lambda^{2}$, in other words, $R(z)$ only 
depends on z. Assuming in addition to Hypothesis 2.1 that

$$
\alpha_{0, \pm} \in \mathbb{C} \backslash\{0\}, \quad \underline{m}=\left(m_{1}, m_{2}\right) \in \mathbb{N}^{2} \backslash\{(0,0)\},
$$

one may write $R(z)$ as

$$
R(z)=\alpha_{0,+}^{2} z^{-2 N_{2}} \prod_{j=1}^{2 N+2}\left(z-z_{j}\right), \quad\left\{z_{j}\right\}_{j=1}^{j=2 N+2} \subset \mathbb{C} \backslash\{0\}, \quad N=N_{1}+N_{2} \in \mathbb{N}_{0}
$$

Moreover, (3.20) also implies

$$
\lim _{z \rightarrow 0} z^{2 N_{2}} R(z)=\alpha_{0,+}^{2} \prod_{j=1}^{2 N+2}\left(-z_{j}\right)=\alpha_{0,-}^{2}
$$

and hence,

$$
\prod_{j=1}^{2 N+2} z_{j}=\frac{\alpha_{0,-}^{2}}{\alpha_{0,+}^{2}}
$$

Equation (3.20) allows us to introduce a hyperelliptic curve $\mathcal{K}_{N}$ of arithmetic genus $N$ (possibly with a singular affine part) defined by

$$
\mathcal{K}_{N}: \mathcal{F}_{N}(z, y)=y^{2}-\alpha_{0,+}^{-2} z^{2 N_{2}} R(z)=y^{2}-\prod_{j=1}^{2 N+2}\left(z-z_{j}\right)=0
$$

The curve $\mathcal{K}_{N}$ can be compactified by joining two points at infinity, $P_{\infty \pm}$, where $P_{\infty+} \neq$ $P_{\infty-}$. For notational simplicity the compactification is also denoted by $\mathcal{K}_{N}$. Points $P$ on $\mathcal{K}_{N} \backslash\left\{P_{\infty+}, P_{\infty-}\right\}$ are represented as pairs $P=(z, y)$, where $y(\cdot)$ is the meromorphic function on $\mathcal{K}_{N}$ satisfying $\mathcal{F}_{N}(z, y)=0$. Here we assume that the zeros $z_{j}$ of $R(z)$ in (3.20) are mutually distinct, which means $z_{j} \neq z_{k}$, for $j \neq k, 1 \leq j, k \leq 2 N+2$. Then the hyperelliptic curve $\mathcal{K}_{N}$ becomes nonsingular. According to the definition of $\mathcal{K}_{N}$, we can lift the roots $\left\{\mu_{j}(n)\right\}$ of $H(n)$ and $\left\{\mu_{j}^{-}(n)\right\}$ of $F(n)$ to $\mathcal{K}_{N}$ by introducing

$$
\begin{aligned}
& \hat{\mu}_{j}\left(n, t_{\underline{m}}\right)=\left(\mu_{j}\left(n, t_{\underline{m}}\right),-\alpha_{0,+}^{-1} \mu_{j}\left(n, t_{\underline{m}}\right)^{N_{2}} G\left(\mu_{j}\left(n, t_{\underline{m}}\right), n, t_{\underline{m}}\right)\right), \\
& \hat{\mu}_{j}^{-}\left(n, t_{\underline{m}}\right)=\left(\mu_{j}^{-}\left(n, t_{\underline{m}}\right), \alpha_{0,+}^{-1} \mu_{j}\left(n, t_{\underline{m}}\right)^{N_{2}} G\left(\mu_{j}^{-}\left(n, t_{\underline{m}}\right), n, t_{\underline{m}}\right)\right),
\end{aligned}
$$

where $j=1, \ldots, N,\left(n, t_{\underline{m}}\right) \in \mathbb{Z} \times \mathbb{R}$. We also introduce the points $P_{0, \pm}$ by

$$
P_{0, \pm}=\left(0, y\left(P_{0, \pm}\right)\right)=\left(0, \pm \frac{\alpha_{0,-}}{\alpha_{0,+}}\right) \in \mathcal{K}_{N}
$$

We emphasize that $P_{0, \pm}$ and $P_{\infty \pm}$ are not necessarily on the same sheet of $\mathcal{K}_{N}$.

Next, we briefly recall our conventions used in connection with divisors on $\mathcal{K}_{N}$. A map, $D: \mathcal{K}_{N} \rightarrow \mathbb{Z}$, is called a divisor on $\mathcal{K}_{N}$ if $D(P) \neq 0$ for only finitely many $P \in \mathcal{K}_{N}$. The set of divisors on $\mathcal{K}_{N}$ is denote by $\operatorname{Div}\left(\mathcal{K}_{N}\right)$. We shall employ the following notation for 
divisors,

$$
\begin{gathered}
D_{Q_{0} \underline{Q}}=D_{\underline{Q}}+D_{Q_{0}}, \quad D_{\underline{Q}}=D_{Q_{1}}+\cdots+D_{Q_{N}}, \\
\underline{Q}=\left\{Q_{1}, \ldots, Q_{N}\right\} \in \operatorname{Sym}^{N}\left(\mathcal{K}_{N}\right), \quad Q_{0} \in \mathcal{K}_{N}, \quad N \in \mathbb{N},
\end{gathered}
$$

where for any $Q \in \mathcal{K}_{N}$,

$$
D_{Q}: \mathcal{K}_{N} \rightarrow \mathbb{N}_{0}, \quad P \mapsto D_{Q}(P)= \begin{cases}1, & \text { for } P=Q, \\ 0, & P \in \mathcal{K}_{N} \backslash\{Q\},\end{cases}
$$

and $\operatorname{Sym}^{N}\left(\mathcal{K}_{N}\right)$ denotes the $N$ th symmetric product of $\mathcal{K}_{N}$.

From (4.5) we know that

$$
\alpha_{0,+}^{2} z^{-2 N_{2}} y^{2}=G^{2}+z F H
$$

that is

$$
\left(\alpha_{0,+} z^{-N_{2}} y+G\right)\left(\alpha_{0,+} z^{-N_{2}} y-G\right)=z F H
$$

then we can define the fundamental meromorphic function $\phi\left(\cdot, n, t_{\underline{m}}\right)$ on $\mathcal{K}_{N}$ by

$$
\begin{aligned}
\phi\left(P, n, t_{\underline{m}}\right)=\frac{\alpha_{0,+} z^{-N_{2}} y-G\left(n, t_{\underline{m}}\right)}{F\left(n, t_{\underline{m}}\right)} & =\frac{z H\left(n, t_{\underline{m}}\right)}{\alpha_{0,+} z^{-N_{2}} y+G\left(n, t_{\underline{m}}\right)}, \\
P & =(z, y) \in \mathcal{K}_{N}, \quad n \in \mathbb{N},
\end{aligned}
$$

with divisor $\left(\phi\left(\cdot, n, t_{\underline{m}}\right)\right)$ of $\phi\left(\cdot, n, t_{\underline{m}}\right)$ given by

$$
\left(\phi\left(\cdot, n, t_{\underline{m}}\right)\right)=D_{P_{0,+\underline{\hat{\nu}}}\left(n, t_{\underline{m}}\right)}-D_{P_{\infty}-\underline{\hat{\mu}}\left(n, t_{\underline{m}}\right)}
$$

using (3.9). Here we abbreviated

$$
\underline{\hat{\mu}}\left(n, t_{\underline{m}}\right)=\left\{\hat{\mu_{1}}\left(n, t_{\underline{m}}\right), \ldots, \hat{\mu}_{N}\left(n, t_{\underline{m}}\right)\right\}, \quad \underline{\hat{\nu}}\left(n, t_{\underline{m}}\right)=\left\{\hat{\nu}_{1}\left(n, t_{\underline{m}}\right), \ldots, \hat{\nu}_{N}\left(n, t_{\underline{m}}\right)\right\} .
$$

In order to straighten out of the corresponding flows, we consider the Riemann surface $\mathcal{K}_{N}$ and equip $\mathcal{K}_{N}$ with canonical basis cycles: $a_{1}, \ldots, a_{N} ; b_{1}, \ldots, b_{N}$, which are independent and have intersection numbers as follows

$$
a_{i} \circ a_{j}=0, \quad b_{i} \circ b_{j}=0, \quad a_{i} \circ b_{j}=\delta_{i j} .
$$

For the present, we will choose our basis as the following set

$$
\tilde{\omega}_{l}=\frac{z^{l-1} d z}{\sqrt{\alpha_{0,+}^{-2} z^{2 N_{2}} R(z)}}, \quad 1 \leq l \leq N,
$$

which are $N$ linearly independent homomorphic differentials on $\mathcal{K}_{N}$. By using the cycles $a_{j}$ and $b_{j}$, the period matrices $A$ and $B$ can be constructed from

$$
A_{i j}=\int_{a_{j}} \tilde{\omega}_{i}, \quad B_{i j}=\int_{b_{j}} \tilde{\omega}_{i} .
$$

It is possible to show that matrices $A$ and $B$ are invertible $[11,13]$. Now we define the matrices $C$ and $\tau$ by $C=A^{-1}, \tau=A^{-1} B$. The matrix $\tau$ can be shown to be symmetric $\left(\tau_{i j}=\tau_{j i}\right)$ and it has positive definite imaginary part $(\operatorname{Im} \tau>0)$. If we normalize $\tilde{\omega}_{l}$ into 
D. Gong $\mathcal{E}$ X. Geng

the new basis $\omega_{j}$

$$
\omega_{j}=\sum_{l=1}^{N} C_{j l} \tilde{\omega}_{l}, \quad 1 \leq j \leq N
$$

then we have

$$
\int_{a_{i}} \omega_{j}=\sum_{l=1}^{N} C_{j l} \int_{a_{i}} \tilde{\omega}_{l}=\delta_{j i}, \quad \int_{b_{i}} \omega_{j}=\tau_{j i} .
$$

Let $\mathcal{T}$ be the lattice generated by $2 N$ vectors $\delta_{j}, \tau_{j}$, where $\delta_{j}=(\underbrace{0, \ldots, 0}_{j-1}, 1, \underbrace{0, \ldots, 0}_{N-j})$ and $\tau_{j}=\tau \delta_{j}$. The complex torus $\mathcal{J}=\mathbb{C}^{N} / \mathcal{T}$ is called Jacobian variety of $\mathcal{K}_{N}$. Now we introduce the Abel map $\mathcal{A}(P): \operatorname{Div}\left(\mathcal{K}_{N}\right) \rightarrow \mathcal{J}$

$$
\mathcal{A}(P)=\int_{P_{0}}^{P} \underline{\omega}, \quad \mathcal{A}\left(\sum n_{k} P_{k}\right)=\sum n_{k} \mathcal{A}\left(P_{k}\right),
$$

where $P, P_{k} \in \mathcal{K}_{N}, \underline{\omega}=\left(\omega_{1}, \ldots, \omega_{N}\right)$. The Riemann theta function is defined as $[11,13]$

$$
\theta(P, D)=\theta(\underline{\Lambda}-\mathcal{A}(P)+\mathcal{A}(D))
$$

with $P \in \mathcal{K}_{N}, D \in \operatorname{Div}\left(\mathcal{K}_{N}\right)$, and $\underline{\Lambda}=\left(\Lambda_{1}, \ldots, \Lambda_{N}\right)$ is defined by

$$
\Lambda_{j}=\frac{1}{2}\left(1+\tau_{j j}\right)-\sum_{\substack{i=1 \\ i \neq j}}^{N} \int_{a_{i}} \omega_{i} \int_{P_{0}}^{P} \omega_{j}, \quad j=1, \ldots, N .
$$

Define the Abel-Jacobi coordinates

$$
\underline{\rho}\left(n, t_{\underline{m}}\right)=\mathcal{A}\left(\sum_{k=1}^{N} P\left(\mu_{k}(n)\right)\right)=\sum_{k=1}^{N} \mathcal{A}\left(P\left(\mu_{k}(n)\right)\right)=\sum_{k=1}^{N} \int_{P_{0}}^{P\left(\mu_{k}(n)\right)} \underline{\omega},
$$

where $P\left(\mu_{k}(n)\right)=\left(\mu_{k}(n), \sqrt{R\left(\mu_{k}(n)\right)}\right)$. The components of the Abel-Jacobi coordinates in (4.15) read

$$
\rho_{j}\left(n, t_{\underline{m}}\right)=\sum_{k=1}^{N} \int_{P_{0}}^{\hat{\mu}_{k}\left(n, t_{\underline{m}}\right)} \omega_{j}=\sum_{k=1}^{N} \sum_{l=1}^{N} C_{j l} \int_{z\left(P_{0}\right)}^{\mu_{k}} \frac{z^{l-1} d z}{\sqrt{\alpha_{0,+}^{-2} z^{2 N_{2}} R(z)}},
$$

where $1 \leq j \leq N$. Without loss of generality, we choose the branch point $P_{0}=\left(z_{j_{0}}, 0\right)$, $j_{0} \in\{1, \ldots, 2 N+2\}$, as a convenient base point on $\mathcal{K}_{N}$, and $z\left(P_{0}\right)$ is its local coordinate. Then according to (4.13) and the definition of Riemann theta function in (4.14), we have

$$
\theta\left(P, D_{\underline{\hat{\mu}}}\left(n, t_{\underline{m}}\right)\right)=\theta\left(\underline{\Lambda}-\mathcal{A}(P)+\underline{\rho}\left(n, t_{\underline{m}}\right)\right) .
$$

From (3.23), we obtain

$$
\partial_{t_{\underline{m}}} \rho_{j}\left(n, t_{\underline{m}}\right)=\sum_{l=1}^{N} \sum_{k=1}^{N} C_{j l} \frac{\mu_{k}^{l-1} \mu_{k, t_{\underline{m}}}}{\sqrt{\alpha_{0,+}^{-2} \mu_{k}^{2 N_{2}} R\left(\mu_{k}\right)}}=\sum_{l=1}^{N} \sum_{k=1}^{N} \frac{\left.\left(-C_{j l}\right) \mu_{k}^{l-1} C^{(\underline{m})}(n)\right|_{z=\mu_{k}}}{\prod_{\substack{i=1 \\ i \neq k}}^{N}\left(\mu_{k}-\mu_{i}\right)} .
$$


Before discussing the following theorem, we start with some elementary results. Let

$$
\left\{z_{j}\right\}_{j=1, \ldots, 2 N+2} \subset \mathbb{C}
$$

for some $N \in N_{0}$ and $\xi \subset \mathbb{C}$, such that $|\xi|<\min \left\{\left|z_{1}\right|^{-1}, \ldots,\left|z_{2 N+2}\right|^{-1}\right\}$, and abbreviate

$$
\underline{z}=\left(z_{1}, \ldots, z_{2 N+2}\right), \quad \underline{z}^{-1}=\left(z_{1}^{-1}, \ldots, z_{2 N+2}^{-1}\right) .
$$

Then

$$
\left(\prod_{j=1}^{2 N+2}\left(1-z_{j} \xi\right)\right)^{-\frac{1}{2}}=\sum_{k=1}^{\infty} \hat{\alpha}_{k}(\underline{z}) \xi^{k}
$$

where

$$
\begin{aligned}
& \hat{\alpha}_{0}(\underline{z})=1, \\
& \hat{\alpha}_{k}(\underline{z})=\sum_{\substack{j_{1}, \ldots, j_{2 N+2}=0 \\
j_{1}+\cdots+j_{2 N+2}=k}}^{k} \frac{\left(2 j_{1}\right) ! \cdots\left(2 j_{2 N+2}\right) !}{2^{2 k}\left(j_{1} !\right)^{2} \cdots\left(j_{2 N+2} !\right)^{2}} z_{1}^{j_{1}} \cdots z_{2 N+2}^{j_{2 N+2}}, \quad k \in \mathbb{N} .
\end{aligned}
$$

The first three coefficients are given explicitly by

$$
\hat{\alpha}_{0}(\underline{z})=1, \quad \hat{\alpha}_{1}(\underline{z})=\frac{1}{2} \sum_{j=1}^{2 N+2} z_{j}, \quad \hat{\alpha}_{2}(\underline{z})=\frac{1}{4} \sum_{\substack{j, k=1 \\ j<k}}^{2 N+2} z_{j} z_{k}+\frac{3}{8} \sum_{j=1}^{2 N+2} z_{j}^{2} .
$$

In a similar way, we have

$$
\left(\prod_{j=1}^{2 N+2}\left(1-z_{j} \xi\right)\right)^{\frac{1}{2}}=\sum_{k=1}^{\infty} \alpha_{k}(\underline{z}) \xi^{k}
$$

where

$$
\begin{aligned}
\alpha_{0}(\underline{z})= & 1, \\
\alpha_{k}(\underline{z})= & \sum_{\substack{j_{1}, \ldots, j_{2 N+2}=0 \\
j_{1}+\cdots+j_{2 N+2}=k}}^{k} \frac{\left(2 j_{1}\right) ! \cdots\left(2 j_{2 N+2}\right) !}{2^{2 k}\left(j_{1} !\right)^{2} \cdots\left(j_{2 N+2} !\right)^{2}\left(2 j_{1}-1\right) \cdots\left(2 j_{2 N+2}-1\right)} \\
& \times z_{1}^{j_{1}} \cdots z_{2 N+2}^{j_{2 N+2}}, \quad k \in \mathbb{N} .
\end{aligned}
$$

The first few coefficients explicitly read

$$
\alpha_{0}(\underline{z})=1, \quad \alpha_{1}(\underline{z})=-\frac{1}{2} \sum_{j=1}^{2 N+2} z_{j}, \quad \alpha_{2}(\underline{z})=\frac{1}{4} \sum_{\substack{j, k=1 \\ j<k}}^{2 N+2} z_{j} z_{k}-\frac{1}{8} \sum_{j=1}^{2 N+2} z_{j}^{2} .
$$

Next, we turn to asymptotic expansions of various quantities in connection with the relativistic Toda hierarchy. We begin with some general results associated with the relativistic Toda hierarchy. Considering a fundamental system of solutions $\Psi_{ \pm}(z, \cdot)=$ $\left(\psi_{1, \pm}(z, \cdot), \psi_{2, \pm}(z, \cdot)\right)^{T}$ of $U(z) \Psi_{ \pm}(z)=\Psi_{ \pm}^{+}(z)$ for $z \in \mathbb{C}$, with $U$ given by (2.1) such 
that

$$
\operatorname{det}\left(\Psi_{-}(z), \Psi_{+}(z)\right) \neq 0
$$

Introducing

$$
\phi_{ \pm}=\frac{\lambda \psi_{2, \pm}}{\psi_{1, \pm}}
$$

then $\phi_{ \pm}$satisfy the Riccati-type equation

$$
-a^{2} \phi_{ \pm} \phi_{ \pm}^{+}-(z-b) \phi_{ \pm}=z, \quad z=\lambda^{2}
$$

and one introduces in addition,

$$
\begin{aligned}
& \check{F}=\frac{2}{\phi_{+}-\phi_{-}}, \\
& \check{G}=-\frac{\phi_{+}+\phi_{-}}{\phi_{+}-\phi_{-}}, \\
& \check{H}=\frac{-2 z^{-1} \phi_{+} \phi_{-}}{\phi_{+}-\phi_{-}} .
\end{aligned}
$$

Using the Riccati-type equation (4.28), one derives the identities

$$
\begin{aligned}
& \check{F}^{+}+a^{2} \check{H}=0, \\
& (z-b) \check{F}^{+}-a^{2}\left(\check{G}^{+}+\check{G}\right)=0, \\
& (z-b) \check{H}+\check{G}^{+}+\check{G}=0, \\
& (z-b) \triangle \check{G}+z a^{2} \check{H}^{+}+z \check{F}=0, \\
& \check{G}^{2}+z \check{F} \check{H}=1 .
\end{aligned}
$$

Moreover, (4.28) also permit one to derive nonlinear difference equations for $\check{G}, \check{F}$, $\check{H}$ separately,

$$
\begin{aligned}
& \left(\left(a^{+}\right)^{2}(z-b)^{2} \check{F}^{+}+z\left(a a^{+}\right)^{2} \check{F}-z a^{4} \check{F}^{++}\right)^{2}-4 a^{2}\left(a^{+}\right)^{4} z(z-b)^{2} \check{F} \check{F}^{+} \\
& \quad=4\left(a a^{+}\right)^{4}(z-b)^{2}, \\
& (z-b)\left(z-b^{-}\right) \check{G}^{2}-z\left(a^{-}\right)^{2}\left(\check{G}^{+}+\check{G}\right)\left(\check{G}+\check{G}^{-}\right)=(z-b)\left(z-b^{-}\right), \\
& \left(z a^{2} \check{H}^{+}-(z-b)^{2} \check{H}-z\left(a^{-}\right)^{2} \check{H}^{-}\right)^{2}-4\left(a^{-}\right)^{2} z(z-b)^{2} \check{H} \check{H}^{-}=4(z-b)^{2} .
\end{aligned}
$$

Next, we assume the existence of the asymptotic expansions of $\check{F}, \check{G}, \check{H}$ as $P \rightarrow P_{\infty \pm}$ and $P \rightarrow P_{0, \pm}$. More precisely, near $\frac{1}{z}=0$ we assume that

$$
\begin{aligned}
& \check{F}(z) \underset{\substack{|z| \rightarrow 0 \\
z \in C_{R}}}{=} \mp \sum_{l=0}^{\infty} \check{f}_{l,+} z^{-l-1}, \quad \check{G}(z) \underset{\substack{|z| \rightarrow 0 \\
z \in C_{R}}}{=} \mp \sum_{l=0}^{\infty} \check{g}_{l,+} z^{-l}, \\
& \check{H}(z) \underset{\substack{|z| \rightarrow 0 \\
z \in C_{R}}}{=} \mp \sum_{l=0}^{\infty} \check{h}_{l,+} z^{-l-1}, \quad l \in \mathbb{N}_{0},
\end{aligned}
$$


for $z$ in some cone $C_{R}$ with apex at $z=0$ and some opening angle in $(0,2 \pi]$, exterior to a disk centered at $z=0$ of Sufficiently large radius $R>0$, for some set of coefficients $\check{f}_{l,+}, \check{g}_{l,+}, \check{h}_{l,+}, l \in \mathbb{N}_{0}$, and the sign depends on whether $P$ tends to $P \rightarrow P_{\infty+}$ or $P \rightarrow P_{\infty-}$. Similarly, near $z=0$ we assume that

$$
\begin{aligned}
& \check{F}(z) \underset{\substack{|z| \rightarrow 0 \\
z \in C_{r}}}{=} \pm \sum_{l=0}^{\infty} \check{f}_{l,-} z^{l}, \quad \check{G}(z) \underset{\substack{|z| \rightarrow 0 \\
z \in C_{r}}}{=} \pm \sum_{l=0}^{\infty} \check{g}_{l,-} z^{l}, \\
& \check{H}(z) \underset{\substack{|z| \rightarrow 0 \\
z \in C_{r}}}{=} \pm \sum_{l=0}^{\infty} \check{h}_{l,-} z^{l}, \quad l \in \mathbb{N}_{0},
\end{aligned}
$$

for $z$ in some cone $C_{r}$ with apex at $z=0$ and some opening angle in $(0,2 \pi]$, interior to a disk centered at $z=0$ of Sufficiently small radius $r>0$, for some set of coefficients $\check{f}_{l,-}, \check{g}_{l,-}, \check{h}_{l,-}, l \in \mathbb{N}_{0}$, and the sign depends on whether $P$ tends to $P \rightarrow P_{0,+}$ or $P \rightarrow P_{0,-}$. Then we can prove the following result.

Theorem 4.1. Suppose $a, b \in \mathbb{C}^{\mathbb{Z}}, a(n) b(n) \neq 0, n \in \mathbb{Z}$, and the existence of the asymptotic expansions (4.32) and (4.33). Then $\check{F}(z), \breve{G}(z), \check{H}(z)$ have the following asymptotic expansions as $P \rightarrow P_{\infty \pm}, z \in C_{R}$, respectively, $P \rightarrow P_{0, \pm}, z \in C_{r}$,

$$
\begin{aligned}
& \check{F}(z) \underset{\substack{|z| \rightarrow 0 \\
z \in C_{R}}}{=} \mp \sum_{l=0}^{\infty} \hat{f}_{l,+} z^{-l-1}, \quad \check{G}(z) \underset{\substack{|z| \rightarrow 0 \\
z \in C_{R}}}{=} \mp \sum_{l=0}^{\infty} \hat{g}_{l,+} z^{-l}, \\
& \check{H}(z) \underset{\substack{|z| \rightarrow 0 \\
z \in C_{R}}}{=} \mp \sum_{l=0}^{\infty} \hat{h}_{l,+} z^{-l-1}, \quad l \in \mathbb{N}_{0},
\end{aligned}
$$

and

$$
\begin{aligned}
& \check{F}(z) \underset{\substack{|z| \rightarrow 0 \\
z \in C_{r}}}{=} \pm \sum_{l=0}^{\infty} \hat{f}_{l,-} z^{l}, \quad \check{G}(z) \underset{\substack{|z| \rightarrow 0 \\
z \in C_{r}}}{=} \pm \sum_{l=0}^{\infty} \hat{g}_{l,-} z^{l}, \\
& \check{H}(z) \underset{\substack{|z| \rightarrow 0 \\
z \in C_{r}}}{=} \pm \sum_{l=0}^{\infty} \hat{h}_{l,-} z^{l}, \quad l \in \mathbb{N}_{0},
\end{aligned}
$$

where $\hat{f}_{l, \pm}, \hat{g}_{l \pm}, \hat{h}_{l, \pm}$ are the homogeneous versions of the coefficients $f_{l, \pm}, g_{l \pm}, h_{l, \pm}$ defined in (3.11). In particular, $\hat{f}_{l, \pm}, \hat{g}_{l \pm}, \hat{h}_{l, \pm}$ can be computed from the nonlinear recursion relations

$$
\begin{aligned}
\hat{f}_{0,+}= & -2\left(a^{-}\right)^{2} \\
\hat{f}_{1,+}= & -2\left(a^{-}\right)^{2}\left(\left(a^{-}\right)^{2}+\left(a^{--}\right)^{2}+b^{-}\right), \\
\hat{f}_{2,+}= & -2\left(a^{-}\right)^{2}\left(\left(a^{-}\right)^{4}+\left(a^{--}\right)^{4}+\left(a a^{-}\right)^{2}+2\left(a^{-} a^{--}\right)^{2}+\left(a^{-}\right)^{2} b+2\left(a^{-}\right)^{2} b^{-}\right. \\
& \left.+\left(a^{--} a^{---}\right)^{2}+2\left(a^{--}\right)^{2} b^{-}+\left(a^{--}\right)^{2} b^{--}+\left(b^{-}\right)^{2}\right),
\end{aligned}
$$


D. Gong $\mathcal{E}$ X. Geng

$$
\begin{aligned}
\hat{f}_{l,+}= & -\frac{1}{4 a^{4}\left(a^{-}\right)^{2}}\left(a^{4} \sum_{m=1}^{l-1} \hat{f}_{m,+} \hat{f}_{l-m,+}-4 a^{4} b^{-} \sum_{m=0}^{l-1} \hat{f}_{m,+} \hat{f}_{l-m-1,+}\right. \\
& -2 a^{4}\left(a^{-}\right)^{2} \sum_{m=0}^{l-1} \hat{f}_{m,+} \hat{f}_{l-m-1,+}^{-}-2\left(a^{-}\right)^{4} a^{2} \sum_{m=0}^{l-1} \hat{f}_{m,+} \hat{f}_{l-m-1,+}^{+} \\
& +\left(a a^{-}\right)^{4} \sum_{m=0}^{l-2} \hat{f}_{m,+}^{-} \hat{f}_{l-m-2,+}^{-}+6 a^{4}\left(b^{-}\right)^{2} \sum_{m=0}^{l-2} \hat{f}_{m,+} \hat{f}_{l-m-2,+} \\
& +\left(a^{-}\right)^{8} \sum_{m=0}^{l-2} \hat{f}_{m,+}^{+} \hat{f}_{l-m-2,+}^{+}+4\left(a^{-}\right)^{2} a^{4} b \sum_{m=0}^{l-2} \hat{f}_{m,+} \hat{f}_{l-m-2,+}^{-} \\
& -2\left(a^{-}\right)^{6} a^{2} \sum_{m=0}^{l-2} \hat{f}_{m,+}^{-} \hat{f}_{l-m-2,+}^{+}+4 a^{2}\left(a^{-}\right)^{4} b^{-} \sum_{m=0}^{l-2} \hat{f}_{m,+} \hat{f}_{l-m-2,+}^{+} \\
& -4 a^{4}\left(b^{-}\right)^{3} \sum_{m=0}^{l-3} \hat{f}_{m,+} \hat{f}_{l-m-3,+}-2\left(a^{-}\right)^{2} a^{4}\left(b^{-}\right)^{2} \sum_{m=0}^{l-3} \hat{f}_{m,+} \hat{f}_{l-m-3,+}^{-} \\
\hat{g}_{l,+} & =\frac{1}{2}\left(\sum_{m=1}^{l-1} \hat{g}_{m,+} \hat{g}_{l-m-1,+}-\left(b+b^{-}\right) \sum_{m=0}^{l-1} \hat{g}_{m,+} \hat{g}_{l-m-1,+}\right. \\
& +b b^{-} \sum_{m=0}^{l-2} \hat{g}_{m,+} \hat{g}_{l-m-2,+}-\left(a^{-}\right)^{2} \sum_{m=0}^{l-1} \hat{g}_{m,+}\left(\hat{g}_{l-m-1,+}^{+}\right. \\
& \left.-2\left(a^{-}\right)^{4} a^{2}\left(b^{-}\right)^{2} \sum_{m=0}^{l-3} \hat{f}_{m,+} \hat{f}_{l-m-3,+}^{+}+a^{4}\left(b^{-}\right)^{4} \sum_{m=0}^{l-1} \hat{f}_{m,+} \hat{f}_{l-m-4,+}\right) \\
\hat{g}_{0,+} & =-1, \quad \hat{g}_{1,+}=-2\left(a^{-}\right)^{4}-\left(a^{-}\right)^{2}\left(a^{2}+\left(a^{-}\right)^{2}+\left(a^{--}\right)^{2}+b+b^{-}\right) \\
& \\
& \\
&
\end{aligned}
$$

$$
\begin{aligned}
\hat{h}_{0,+}= & 2, \quad \hat{h}_{1,+}=2\left(a^{2}+\left(a^{-}\right)^{2}+b\right), \\
\hat{h}_{2,+}= & 2\left(a^{4}+\left(a^{-}\right)^{4}+\left(a a^{+}\right)^{2}+2\left(a a^{-}\right)^{2}+a^{2} b^{+}+2 a^{2} b+\left(a^{-} a^{--}\right)^{2}\right. \\
& \left.+2\left(a^{-}\right)^{2} b+\left(a^{-}\right)^{2} b^{-}+b^{2}\right), \\
\hat{h}_{l,+}= & -\frac{1}{4}\left(\sum_{m=1}^{l-1} \hat{h}_{m,+} \hat{h}_{l-m,+}-4 b \sum_{m=0}^{l-1} \hat{h}_{m,+} \hat{h}_{l-m-1,+}-2 a^{2} \sum_{m=0}^{l-1} \hat{h}_{m,+}^{+} \hat{h}_{l-m-1,+}\right. \\
& -2\left(a^{-}\right)^{2} \sum_{m=0}^{l-1} \hat{h}_{m,+} \hat{h}_{l-m-1,+}+a^{4} \sum_{m=0}^{l-2} \hat{h}_{m,+}^{+} \hat{h}_{l-m-2,+}^{+}+6 b^{2} \sum_{m=0}^{l-2} \hat{h}_{m,+} \hat{h}_{l-m-2,+}
\end{aligned}
$$




$$
\begin{aligned}
& +\left(a^{-}\right)^{4} \sum_{m=0}^{l-2} \hat{h}_{m,+}^{-} \hat{h}_{l-m-2,+}^{-}+4 a^{2} b \sum_{m=0}^{l-2} \hat{h}_{m,+}^{+} \hat{h}_{l-m-2,+}-2\left(a a^{-}\right)^{2} \sum_{m=0}^{l-2} \hat{h}_{m,+}^{+} \hat{h}_{l-m-2,+}^{-} \\
& +4\left(a^{-}\right)^{2} b \sum_{m=0}^{l-2} \hat{h}_{m,+} \hat{h}_{l-m-2,+}^{-}-4 b^{3} \sum_{m=0}^{l-3} \hat{h}_{m,+} \hat{h}_{l-m-3,+}-2 a^{2} b^{2} \sum_{m=0}^{l-3} \hat{h}_{m,+}^{+} \hat{h}_{l-m-3,+} \\
& \left.-2\left(a^{-}\right)^{2} b^{2} \sum_{m=0}^{l-3} \hat{h}_{m,+} \hat{h}_{l-m-3,+}^{-}+b^{4} \sum_{m=0}^{l-4} \hat{h}_{m,+} \hat{h}_{l-m-4,+}\right), \quad l \geq 3,
\end{aligned}
$$

$$
\begin{aligned}
& \hat{f}_{0,-}=-\frac{2\left(a^{-}\right)^{2}}{b^{-}} \\
& \hat{f}_{1,-}=-\frac{2\left(a^{-}\right)^{2}}{\left(b^{-}\right)^{2}}\left(1+\frac{\left(a^{-}\right)^{2}}{b}+\frac{\left(a^{--}\right)^{2}}{b^{--}}\right) \\
& \hat{f}_{2,-}=-\frac{2\left(a^{-}\right)^{2}}{\left(b^{-}\right)^{3}}\left(1+\frac{\left(a^{-}\right)^{4}}{b^{2}}+\frac{\left(a^{--}\right)^{4}}{\left(b^{--}\right)^{2}}+\frac{2\left(a^{-}\right)^{2}}{b}+\frac{2\left(a^{--}\right)^{2}}{b^{--}}+\frac{2\left(a^{-} a^{--}\right)^{2}}{b b^{--}}+\frac{\left(a^{-}\right)^{2} b^{-}}{b^{2}}\right. \\
& \left.+\frac{\left(a^{--}\right)^{2} b^{-}}{\left(b^{--}\right)^{2}}+\frac{\left(a a^{-}\right)^{2} b^{-}}{b^{2} b^{+}}+\frac{\left(a^{--} a^{---}\right)^{2} b^{-}}{\left(b^{--}\right)^{2} b^{---}}\right) \text {. } \\
& \hat{f}_{l,-}=-\frac{b^{-}}{4\left(a^{-}\right)^{2}\left(a^{+}\right)^{4} b^{4}}\left(\left(a^{+}\right)^{4} \sum_{m=0}^{l-4} \hat{f}_{m,-}^{+} \hat{f}_{l-m-4,-}^{+}-4\left(a^{+}\right)^{4} b \sum_{m=1}^{l-3} \hat{f}_{m,-}^{+} \hat{f}_{l-m-3,-}^{+}\right. \\
& -2 a^{2}\left(a^{+}\right)^{4} \sum_{m=0}^{l-3} \hat{f}_{m,-}^{+} \hat{f}_{l-m-3,-}-2 a^{4}\left(a^{+}\right)^{2} \sum_{m=0}^{l-3} \hat{f}_{m,-}^{+} \hat{f}_{l-m-3,-}^{++} \\
& +\left(a a^{+}\right)^{4} \sum_{m=0}^{l-2} \hat{f}_{m,-} \hat{f}_{l-m-2,-}+6\left(a^{+}\right)^{4} b^{2} \sum_{m=0}^{l-2} \hat{f}_{m,-}^{+} \hat{f}_{l-m-2,-}^{+} \\
& +a^{8} \sum_{m=0}^{l-2} \hat{f}_{m,-}^{++} \hat{f}_{l-m-2,-}^{++}+4 a^{2}\left(a^{+}\right)^{4} b \sum_{m=0}^{l-2} \hat{f}_{m,-}^{+} \hat{f}_{l-m-2,-} \\
& -2 a^{6}\left(a^{+}\right)^{2} \sum_{m=0}^{l-2} \hat{f}_{m,-} \hat{f}_{l-m-2,-}^{++}+4 a^{4}\left(a^{+}\right)^{2} b \sum_{m=0}^{l-2} \hat{f}_{m,-}^{+} \hat{f}_{l-m-2,-}^{++} \\
& -4\left(a^{+}\right)^{4} b^{3} \sum_{m=0}^{l-1} \hat{f}_{m,-}^{+} \hat{f}_{l-m-1,-}^{+}-2 a^{2}\left(a^{+}\right)^{4} b^{2} \sum_{m=0}^{l-1} \hat{f}_{m,-}^{+} \hat{f}_{l-m-1,-} \\
& \left.-2 a^{4}\left(a^{+}\right)^{2} b^{2} \sum_{m=0}^{l-1} \hat{f}_{m,-}^{+} \hat{f}_{l-m-1,-}^{++}+\left(a^{+}\right)^{4} b^{4} \sum_{m=1}^{l-1} \hat{f}_{m,-}^{+} \hat{f}_{l-m,-}^{+}\right), \quad l \geq 3,
\end{aligned}
$$$$
\hat{g}_{0,-}(n)=1, \quad \hat{g}_{1,-}(n)=\frac{2\left(a^{-}\right)^{2}}{b b^{-}},
$$$$
\hat{g}_{2,-}=\frac{4\left(a^{-}\right)^{2}\left(b+b^{-}\right)}{\left(b b^{-}\right)^{2}}-\frac{4\left(a a^{-}\right)^{2}}{b^{+} b^{2} b^{-}}-\frac{4\left(a^{-} a^{--}\right)^{2}}{b\left(b^{-}\right)^{2} b^{--}},
$$ 


$$
\begin{aligned}
& \hat{g}_{l,-}=\frac{1}{2 b b^{-}}\left(-\sum_{m=0}^{l-2} \hat{g}_{m,-} \hat{g}_{l-m-2,-}+\left(b+b^{-}\right) \sum_{m=0}^{l-1} \hat{g}_{m,-} \hat{g}_{l-m-1,-}\right. \\
& +b b^{-} \sum_{m=1}^{l-1} \hat{g}_{m,-} \hat{g}_{l-m,+} \\
& +\left(a^{-}\right)^{2} \sum_{m=0}^{l-1} \hat{g}_{m,-}\left(\hat{g}_{l-m-1,-}^{+}+\hat{g}_{l-m-1,-}+\hat{g}_{l-m-1,-}^{-}\right) \\
& \left.+\left(a^{-}\right)^{2} \sum_{m=0}^{l-1} \hat{g}_{m,-}^{+} \hat{g}_{l-m-1,-}^{-}\right), \quad l \geq 3, \\
& \hat{h}_{0,-}=\frac{2}{b}, \quad \hat{h}_{1,-}=\frac{2}{b^{2}}\left(1+\frac{a^{2}}{b^{+}}+\frac{\left(a^{-}\right)^{2}}{b^{-}}\right), \\
& \hat{h}_{2,-}=\frac{2}{b^{3}}\left(1+\frac{a^{4}}{\left(b^{+}\right)^{2}}+\frac{\left(a^{-}\right)^{4}}{\left(b^{-}\right)^{2}}+\frac{2 a^{2}}{b^{+}}+\frac{2\left(a^{-}\right)^{2}}{b^{-}}+\frac{2\left(a a^{-}\right)^{2}}{b^{+} b^{-}}+\frac{(a)^{2} b}{\left(b^{+}\right)^{2}}+\frac{\left(a^{-}\right)^{2} b}{\left(b^{-}\right)^{2}}\right. \\
& \left.+\frac{\left(a a^{+}\right)^{2} b}{\left(b^{+}\right)^{2} b^{++}}+\frac{\left(a^{-} a^{--}\right)^{2} b}{\left(b^{-}\right)^{2} b^{--}}\right) . \\
& \hat{h}_{l,-}=-\frac{1}{4 b^{3}}\left(\sum_{m=0}^{l-4} \hat{h}_{m,-} \hat{h}_{l-m-4,-}-4 b \sum_{m=1}^{l-3} \hat{h}_{m,-} \hat{h}_{l-m-3,-}-2 a^{2} \sum_{m=0}^{l-3} \hat{h}_{m,-}^{+} \hat{h}_{l-m-3,-}\right. \\
& -2\left(a^{-}\right)^{2} \sum_{m=0}^{l-3} \hat{h}_{m,-} \hat{h}_{l-m-3,-}^{-}+a^{4} \sum_{m=0}^{l-2} \hat{h}_{m,-}^{+} \hat{h}_{l-m-2,-}^{+}+6 b^{2} \sum_{m=0}^{l-2} \hat{h}_{m,-} \hat{h}_{l-m-2,-} \\
& +\left(a^{-}\right)^{2} \sum_{m=0}^{l-2} \hat{h}_{m,-}^{-} \hat{h}_{l-m-2,-}^{-}+4 a^{2} b \sum_{m=0}^{l-2} \hat{h}_{m,-}^{+} \hat{h}_{l-m-2,-}-2\left(a a^{-}\right)^{2} \sum_{m=0}^{l-2} \hat{h}_{m,-}^{+} \hat{h}_{l-m-2,-}^{-} \\
& +4\left(a^{-}\right)^{2} b \sum_{m=0}^{l-2} \hat{h}_{m,-} \hat{h}_{l-m-2,-}^{-}-4 b^{3} \sum_{m=0}^{l-1} \hat{h}_{m,-} \hat{h}_{l-m-1,-}-2 a^{2} b^{2} \sum_{m=0}^{l-1} \hat{h}_{m,-}^{+} \hat{h}_{l-m-1,-} \\
& \left.-2\left(a^{-}\right)^{2} b^{2} \sum_{m=0}^{l-1} \hat{h}_{m,-} \hat{h}_{l-m-1,-}+b^{4} \sum_{m=1}^{l-1} \hat{h}_{m,-} \hat{h}_{l-m,-}\right), \quad l \geq 3 .
\end{aligned}
$$

Proof. A direct calculation shows that $\hat{f}_{l, \pm}$ satisfies (4.36) and (4.39), $\hat{g}_{l, \pm}$ satisfies (4.37) and (4.40), $\hat{h}_{l, \pm}$ satisfies (4.38) and (4.41). In order to prove (4.34) and (4.35), one only need to prove $\check{f}_{l, \pm}=\hat{f}_{l, \pm}, \check{g}_{l, \pm}=\hat{g}_{l, \pm}, \check{h}_{l, \pm}=\hat{h}_{l, \pm}, l \in \mathbb{N}_{0}$. We first consider the expansions (4.34) near $\frac{1}{z}=0$ and the nonlinear recursions (4.36)-(4.38) in detail. Inserting expansions (4.32) for $\check{F}$ into (4.29), expansions (4.32) for $\check{G}$ into (4.30), expansions (4.32) for $\check{H}$ into (4.31), then yields the nonlinear recursion relations $(4.36)-(4.38)$, but with $\hat{f}_{l,+}, \hat{g}_{l,+}, \hat{h}_{l,+}$ replaced by $\check{f}_{l,+}, \check{g}_{l,+}, \check{h}_{l,+}$. The signs of $\check{f}_{0,+}, \check{g}_{0,+}, \check{h}_{0,+}$ have been chosen such that the coefficients $\check{f}_{0,+}=-2 a^{2}(n-1), \check{g}_{0,+}=-1, \check{h}_{0,+}=2 . \check{f}_{0,+}, \check{g}_{0,+}, \check{h}_{0,+}$ are consistent with 
(3.11) for $\alpha_{0,+}=1$. Thus, one concludes that

$$
\check{f}_{l,+}=f_{l,+}, \quad \check{g}_{l,+}=g_{l,+}, \quad \check{h}_{l,+}=h_{l,+}, \quad l \in \mathbb{N}_{0},
$$

for certain values of the summation $\alpha_{l,+}$. In order to prove that

$$
\check{f}_{l,+}=\hat{f}_{l,+}, \quad \check{g}_{l,+}=\hat{g}_{l,+}, \quad \check{h}_{l,+}=\hat{h}_{l,+}, \quad l \in \mathbb{N}_{0},
$$

and hence all $\alpha_{l,+}, l \in \mathbb{N}$ vanish, we now depend on the notion of degree as introduced in Remark 3.1. To this effect, we recall that

$$
\operatorname{deg}\left(\hat{f}_{l,+}\right)=2 l+2, \quad \operatorname{deg}\left(\hat{g}_{l,+}\right)=2 l, \quad \operatorname{deg}\left(\hat{h}_{l,+}\right)=2 l, \quad l \in \mathbb{N}_{0}
$$

Similarly, the nonlinear recursion relations (4.36)-(4.38) yield inductively that $\operatorname{deg}\left(\check{f}_{l,+}\right)=$ $2 l+2, \operatorname{deg}\left(\check{g}_{l,+}\right)=2 l, \operatorname{deg}\left(\check{h}_{l,+}\right)=2 l, l \in \mathbb{N}_{0}$. Hence one concludes

$$
\check{f}_{l,+}=\hat{f}_{l,+}, \quad \check{g}_{l,+}=\hat{g}_{l,+}, \quad \check{h}_{l,+}=\hat{h}_{l,+}, \quad l \in \mathbb{N}_{0} .
$$

In a similar way, we can prove that

$$
\check{f}_{l,-}=\hat{f}_{l,-}, \quad \check{g}_{l,-}=\hat{g}_{l,-}, \quad \check{h}_{l,-}=\hat{h}_{l,-}, \quad l \in \mathbb{N}_{0} .
$$

Given this general result on asymptotic expansions for Laurent polynomial associated with relativistic Toda hierarchy, we now specialize to the algebra-geometric case at hand. We recall our convention $y(P)=\mp\left(\zeta^{-N-1}+O\left(\zeta^{-N}\right)\right)$ for $P \rightarrow P_{\infty \pm}$ (where $\zeta=\frac{1}{z}$ ) and $y(P)= \pm \frac{\alpha_{0,-}}{\alpha_{0,+}}+O(\zeta)$ for $P \rightarrow P_{0, \pm}($ where $\zeta=z)$.

Theorem 4.2. Assume $a, b \in \mathbb{C}^{\mathbb{Z}}, a(n) b(n) \neq 0, n \in \mathbb{Z}$, and suppose $P=(z, y) \in$ $\mathcal{K}_{N} \backslash\left\{P_{\infty \pm}, P_{0, \pm}\right\}$. Then $z^{N_{2}} G / y, z^{N_{2}} F / y, z^{N_{2}} H / y$ have the following convergent expansions as $P \rightarrow P_{\infty \pm}$,

$$
\begin{gathered}
\frac{z^{N_{2}}}{\alpha_{0,+}} \frac{G(z)}{y} \underset{\zeta \rightarrow 0}{=} \mp \sum_{l=0}^{\infty} \hat{g}_{l,+} \zeta^{l}, \quad \frac{z^{N_{2}}}{\alpha_{0,+}} \frac{F(z)}{y} \underset{\zeta \rightarrow 0}{=} \mp \sum_{l=0}^{\infty} \hat{f}_{l,+} \zeta^{l+1}, \\
\frac{z^{N_{2}}}{\alpha_{0},+} \frac{H(z)}{y} \underset{\zeta \rightarrow 0}{=} \mp \sum_{l=0}^{\infty} \hat{h}_{l,+} \zeta^{l+1},
\end{gathered}
$$

and as $P \rightarrow P_{0, \pm}$

$$
\begin{gathered}
\frac{z^{N_{2}}}{\alpha_{0,+}} \frac{G(z)}{y} \underset{\zeta \rightarrow 0}{=} \pm \sum_{l=0}^{\infty} \hat{g}_{l,-} \zeta^{l}, \quad \frac{z^{N_{2}}}{\alpha_{0,+}} \frac{F(z)}{y} \underset{\zeta \rightarrow 0}{=} \pm \sum_{l=1}^{\infty} \hat{f}_{l,-} \zeta^{l}, \\
\frac{z^{N_{2}}}{\alpha_{0,+}} \frac{H(z)}{y} \underset{\zeta \rightarrow 0}{=} \pm \sum_{l=1}^{\infty} \hat{h}_{l,-} \zeta^{l},
\end{gathered}
$$

where $\zeta=z^{-1}$ (respectively, $\zeta=z$ ) is the local coordinate near $P_{\infty \pm}$ (respectively, $P \rightarrow$ $\left.P_{0, \pm}\right)$, and $\hat{f}_{l, \pm}, \hat{g}_{l \pm}, \hat{h}_{l, \pm}$ are the homogeneous versions of the coefficients $f_{l, \pm}, g_{l \pm}, h_{l, \pm}$ 
as introduced in (3.11). Moreover, one infers for the $z_{j}$-dependent summation constants $\alpha_{l,+}, l=0, \ldots, N_{1}$ and $\alpha_{l,-}, l=0, \ldots, N_{2}-1$ in $G(z), F(z), H(z)$ that

$$
\alpha_{k,+}=\alpha_{0,+} \alpha_{k}(\underline{z}), \quad 0 \leq k \leq N_{1} ; \quad \alpha_{k,-}=\alpha_{0,-} \alpha_{k}\left(\underline{z}^{-1}\right), \quad 0 \leq k \leq N_{2}-1,
$$

where $\underline{z}=\left(z_{1}, \ldots, z_{2 N+2}\right), \underline{z}^{-1}=\left(z_{1}^{-1}, \ldots, z_{2 N+2}^{-1}\right)$,

$$
\begin{aligned}
& \alpha_{0}\left(\underline{z}^{ \pm 1}\right)=1, \\
& \alpha_{k}\left(\underline{z}^{ \pm 1}\right)=\sum_{\substack{j_{1}, \ldots, j_{2 N+2}=0 \\
j_{1}+\cdots+j_{2 N+2}=k}}^{k} \frac{\left(2 j_{1}\right) ! \cdots\left(2 j_{2 N+2}\right) ! z_{1}^{ \pm j_{1}} \cdots z_{2 N+2}^{ \pm j_{2 N+2}}}{2^{2 k}\left(j_{1} !\right)^{2} \cdots\left(j_{2 N+2} !\right)^{2}\left(2 j_{1}-1\right) \cdots\left(2 j_{2 N+2}-1\right)}, \quad k \in \mathbb{N} .
\end{aligned}
$$

In addition, one has the following relations between the homogeneous and nonhomogeneous recursion coefficients:

$$
\begin{aligned}
& f_{l, \pm}=\alpha_{0, \pm} \sum_{k=0}^{l} \alpha_{l-k}\left(\underline{z}^{ \pm 1}\right) \hat{f}_{k, \pm}, \quad l=0, \ldots, N_{1+\delta_{ \pm}}-\delta_{ \pm}, \\
& g_{l, \pm}=\alpha_{0, \pm} \sum_{k=0}^{l} \alpha_{l-k}\left(\underline{z}^{ \pm 1}\right) \hat{g}_{k, \pm}, \quad l=0, \ldots, N_{1+\delta_{ \pm}} \pm 1, \\
& h_{l, \pm}=\alpha_{0, \pm} \sum_{k=0}^{l} \alpha_{l-k}\left(\underline{z}^{ \pm 1}\right) \hat{h}_{k, \pm}, \quad l=0, \ldots, N_{1+\delta_{ \pm}}-\delta_{ \pm}, \\
& \alpha_{0, \pm} \hat{f}_{l, \pm}=\sum_{k=0}^{l} \hat{\alpha}_{l-k}\left(\underline{z}^{ \pm 1}\right) f_{k, \pm}, \quad l=0, \ldots, N_{1+\delta_{ \pm}}-\delta_{ \pm}, \\
& \alpha_{0, \pm} \hat{g}_{l, \pm}=\sum_{k=0}^{l} \hat{\alpha}_{l-k}\left(\underline{z}^{ \pm 1}\right) g_{k, \pm}, \quad l=0, \ldots, N_{1+\delta_{ \pm}} \pm 1, \\
& \alpha_{0, \pm} \hat{h}_{l, \pm}=\sum_{k=0}^{l} \hat{\alpha}_{l-k}\left(\underline{z}^{ \pm 1}\right) h_{k, \pm}, \quad l=0, \ldots, N_{1+\delta_{ \pm}}-\delta_{ \pm},
\end{aligned}
$$

where

$$
\hat{\alpha}_{0}\left(\underline{z}^{ \pm 1}\right)=1, \quad \hat{\alpha}_{k}\left(\underline{z}^{ \pm 1}\right)=\sum_{\substack{j_{1}, \ldots, j_{2 N+2}=0 \\ j_{1}+\cdots+j_{2 N+2}=k}}^{k} \frac{\left(2 j_{1}\right) ! \cdots\left(2 j_{2 N+2}\right) ! z_{1}^{ \pm j_{1}} \cdots z_{2 N+2}^{ \pm j_{2 N+2}}}{2^{2 k}\left(j_{1} !\right)^{2} \cdots\left(j_{2 N+2} !\right)^{2}}, \quad k \in \mathbb{N} .
$$

Here we used the convention

$$
\delta_{ \pm}= \begin{cases}0, & + \\ 1, & -\end{cases}
$$

Proof. Now, we introduce the holomorphic sheet exchange map

$$
*: \mathcal{K}_{N} \rightarrow \mathcal{K}_{N}, \quad P=(z, y) \rightarrow P^{*}=(z,-y), \quad y\left(P^{*}\right)=-y(P), \quad P, P^{*} \in \mathcal{K}_{N} .
$$

Identifying $\phi_{+}(z, \cdot)$ with $\phi(P, \cdot)$ and $\phi_{-}(z, \cdot)$ with $\phi\left(P^{*}, \cdot\right)$, then $\phi(P, \cdot)$ and $\phi\left(P^{*}, \cdot\right)$ satisfy the Riccati-type equation (4.24). From the definition of meromorphic function $\phi$ in (4.9) 
and the expressions (4.25)-(4.29), we have

$$
\begin{aligned}
& \check{F}=\frac{2}{\phi(P)-\phi\left(P^{*}\right)}=\frac{z^{N_{2}}}{\alpha_{0,+}} \frac{F(z)}{y}, \\
& \check{G}=-\frac{\phi(P)+\phi\left(P^{*}\right)}{\phi(P)-\phi\left(P^{*}\right)}=\frac{z^{N_{2}}}{\alpha_{0,+}} \frac{G(z)}{y}, \\
& \check{H}=\frac{-2 z^{-1} \phi(P) \phi\left(P^{*}\right)}{\phi(P)-\phi\left(P^{*}\right)}=\frac{z^{N_{2}}}{\alpha_{0,+}} \frac{H(z)}{y} .
\end{aligned}
$$

Moreover, as $P \rightarrow P_{\infty \pm}$, one obtains the following expansions using (3.11):

$$
\begin{aligned}
& \frac{z^{N_{2}}}{\alpha_{0,+}} \frac{F(z)}{y} \underset{\zeta \rightarrow 0}{=} \mp \frac{\zeta}{\alpha_{0,+}}\left(\sum_{k=0}^{\infty} \hat{\alpha}_{k}(\underline{\lambda}) \zeta^{k}\right)\left(\sum_{j=0}^{N_{2}-1} f_{N_{2}-1-j,-} \zeta^{N_{1}+j}+\sum_{j=0}^{N_{1}} f_{N_{1}-j,+} \zeta^{N_{1}-j}\right) \\
& \underset{\zeta \rightarrow 0}{=} \mp \sum_{l=0}^{\infty} \hat{f}_{l,+} \zeta^{l+1} \\
& \frac{z^{N_{2}}}{\alpha_{0,+}} \frac{G(z)}{y} \underset{\zeta \rightarrow 0}{=} \mp \frac{\zeta}{\alpha_{0,+}}\left(\sum_{k=0}^{\infty} \hat{\alpha}_{k}(\underline{z}) \zeta^{k}\right)\left(\sum_{j=0}^{N_{2}-1} g_{N_{2}-j,-} \zeta^{N_{1}+j}+\sum_{j=0}^{N_{1}+1} g_{N_{1}+1-j,+} \zeta^{N_{1}-j}\right) \\
& \underset{\zeta \rightarrow 0}{=} \mp \sum_{l=0}^{\infty} \hat{g}_{l,+} \zeta^{l} \\
& \frac{z^{N_{2}}}{\alpha_{0,+}} \frac{H(z)}{y} \underset{\zeta \rightarrow 0}{=} \mp \frac{\zeta}{\alpha_{0,+}}\left(\sum_{k=0}^{\infty} \hat{\alpha}_{k}(\underline{z}) \zeta^{k}\right)\left(\sum_{j=0}^{N_{2}-1} h_{N_{2}-1-j,-} \zeta^{N_{1}+j}+\sum_{j=0}^{N_{1}} h_{N_{1}-j,+} \zeta^{N_{1}-j}\right) \\
& \underset{\zeta \rightarrow 0}{=} \mp \sum_{l=0}^{\infty} \hat{h}_{l,+} \zeta^{l+1} .
\end{aligned}
$$

This implies (4.42) as $P \rightarrow P_{\infty \pm}$. Similarly, as $P \rightarrow P_{0, \pm}$,

$$
\begin{aligned}
& \frac{z^{N_{2}}}{\alpha_{0,+}} \frac{F(z)}{y} \underset{\zeta \rightarrow 0}{=} \pm \frac{1}{\alpha_{0,-}}\left(\sum_{k=0}^{\infty} \hat{\alpha}_{k}\left(\underline{z}^{-1}\right) \zeta^{k}\right)\left(\sum_{j=0}^{N_{2}-1} f_{N_{2}-1-j,-} \zeta^{N_{1}+j}+\sum_{j=0}^{N_{1}} f_{N_{1}-j,+} \zeta^{N_{1}-j}\right) \\
& \underset{\zeta \rightarrow 0}{=} \pm \sum_{l=0}^{\infty} \hat{f}_{l,-} \zeta^{l} \\
& \frac{z^{N_{2}}}{\alpha_{0,+}} \frac{G(z)}{y} \underset{\zeta \rightarrow 0}{=} \pm \frac{1}{\alpha_{0,-}}\left(\sum_{k=0}^{\infty} \hat{\alpha}_{k}\left(\underline{z}^{-1}\right) \zeta^{k}\right)\left(\sum_{j=0}^{N_{2}-1} g_{N_{2}-j,-} \zeta^{N_{1}+j}+\sum_{j=0}^{N_{1}+1} g_{N_{1}+1-j,+} \zeta^{N_{1}+1-j}\right) \\
& \underset{\zeta \rightarrow 0}{=} \pm \sum_{l=0}^{\infty} \hat{g}_{l,-} \zeta^{l}
\end{aligned}
$$




$$
\begin{aligned}
\frac{z^{N_{2}}}{\alpha_{0,+}} \frac{H(z)}{y} & \underset{\zeta \rightarrow 0}{=} \pm \frac{1}{\alpha_{0,-}}\left(\sum_{k=0}^{\infty} \hat{\alpha}_{k}\left(\underline{z}^{-1}\right) \zeta^{k}\right)\left(\sum_{j=0}^{N_{2}-1} h_{N_{2}-1-j,-} \zeta^{N_{1}+j}+\sum_{j=0}^{N_{1}} h_{N_{1}-j,+} \zeta^{N_{1}-j}\right) \\
& = \pm \sum_{\substack{\zeta \rightarrow 0 \\
=}}^{\infty} \hat{h}_{l,-} \zeta^{l} .
\end{aligned}
$$

Thus, (4.43) holds as $P \rightarrow P_{0, \pm}$.

Next, by comparing powers of $\zeta$ in the second and third term of (4.56) and (4.59), respectively, formula (4.49) follows. Similarly, we can get formula (4.50) and formula (4.51).

Finally, multiplying (4.18) and (4.21) and comparing coefficients of $\zeta^{k}$, one finds $\sum_{l=0}^{k} \alpha_{l}\left(\underline{z}^{ \pm 1}\right) \hat{\alpha}_{k-l}\left(\underline{z}^{ \pm 1}\right)=\delta_{k, 0}$, then, one computes

$$
\alpha_{0, \pm} \sum_{k=0}^{l} \alpha_{l-k}\left(\underline{z}^{ \pm 1}\right) \hat{f}_{k, \pm}=\sum_{k=0}^{l} \alpha_{l-k}\left(\underline{z}^{ \pm 1}\right) \sum_{s=0}^{k} \hat{\alpha}_{k-s}\left(\underline{z}^{ \pm 1}\right) f_{s, \pm}=f_{l, \pm}, \quad k \in \mathbb{N}_{0} .
$$

Hence (4.46) holds and (4.44) can be proved using (3.14). The proofs of expansions (4.47) and (4.48) follow precisely the same strategy and are hence omitted.

Assuming $N \in \mathbb{N}$ to be fixed and introducing

$$
\begin{aligned}
& \mathbf{S}_{k}=\left\{\underline{l}=\left(l_{1}, \ldots, l_{k}\right) \in \mathbb{N}^{k} \mid 1 \leq l_{1}<\cdots<l_{k} \leq N\right\}, \quad k=1, \ldots, N, \\
& \mathbf{I}_{k}^{(j)}=\left\{\underline{l}=\left(l_{1}, \ldots, l_{k}\right) \in \mathbf{S}_{k} \mid l_{i} \neq j ; i=1, \ldots, k\right\}, \quad k=1, \ldots, N-1, j=1, \ldots, N,
\end{aligned}
$$

one defines the symmetric functions

$$
\begin{aligned}
& \Psi_{0}(\underline{\mu})=1, \quad \boldsymbol{\Psi}_{k}(\underline{\mu})=(-1)^{k} \sum_{\underline{l} \in \mathbf{S}_{k}} \mu_{l_{1}} \cdots \mu_{l_{k}}, \quad k=1, \ldots, N, \\
& \mathbf{T}_{k}^{j}(\underline{\mu})=(-1)^{k} \sum_{\underline{l} \in \mathbf{I}_{k}^{(j)}} \mu_{l_{1}} \cdots \mu_{l_{k}}, \quad k=1, \ldots, N-1, \quad j=1, \ldots, N, \\
& \mathbf{T}_{0}^{j}(\underline{\mu})=1, \quad \mathbf{T}_{N}^{j}(\underline{\mu})=0, \quad j=1, \ldots, N,
\end{aligned}
$$

where $\underline{\mu}=\left(\mu_{1}, \ldots, \mu_{N}\right) \in \mathbb{C}^{N}$. Introducing

$$
\widetilde{H}(z)=\prod_{j=1}^{N}\left(z-\mu_{j}\right)=\sum_{l=0}^{N} \boldsymbol{\Psi}_{l}(\underline{\mu}) z^{N-l}
$$

one infers

$$
\widetilde{H}_{z}\left(\mu_{k}\right)=\prod_{\substack{j=1 \\ j \neq k}}^{N}\left(\mu_{k}-\mu_{j}\right)
$$

According to Lagrange interpolation theorem, we have the following important facts (these theorems were proven in detail in [5, Theorem D.1, Lemmas D.2 and D.3]). 
Theorem 4.3. Suppose that $\mu_{1}, \ldots, \mu_{N}$ are $N$ distinct complex numbers. Then,

$$
\begin{aligned}
\sum_{j=1}^{N} \frac{\mu_{j}^{l-1}}{\widetilde{H}_{z}\left(\mu_{j}\right)} \mathbf{T}_{k}^{j}(\underline{\mu})= & \delta_{l, N-k}-\mathbf{\Psi}_{k+l}(\underline{\mu}) \delta_{l, N+1}, \\
& l=1, \ldots, N+1, \quad k=0, \ldots, N-1 .
\end{aligned}
$$

Theorem 4.4. Suppose that $\mu_{1}, \ldots, \mu_{N}$ are $N$ distinct complex numbers. Then,

$$
\begin{gathered}
\mathbf{\Psi}_{k+l}(\underline{\mu})+\mu_{j} \mathbf{T}_{k}^{j}(\underline{\mu})=\mathbf{T}_{k+1}^{j}(\underline{\mu}), \quad j=1, \ldots, N, \quad k=0, \ldots, N-1, \\
\sum_{l=0}^{k} \boldsymbol{\Psi}_{k-l}(\underline{\mu}) \mu_{j}^{l}=\mathbf{T}_{k}^{j}(\underline{\mu}), \quad j=1, \ldots, N, \quad k=0, \ldots, N .
\end{gathered}
$$

Assume that $\mu_{j} \neq \mu_{j^{\prime}}$ for $j \neq j^{\prime}$, we introduce the $N \times N$ matrix $\mathbf{B}_{N}(\underline{\mu})$ by

$$
\mathbf{B}_{1}(\underline{\mu})=1, \quad \mathbf{B}_{N}(\underline{\mu})=\left(\frac{\mu_{k}^{j-1}}{\prod_{\substack{i=1 \\ i \neq k}}^{N}\left(\mu_{k}-\mu_{i}\right)}\right)_{j, k=1}^{N}
$$

where $\underline{\mu}=\left(\mu_{1}, \ldots, \mu_{N}\right) \in \mathbb{C}^{N}$.

Theorem 4.5. Suppose that $\mu_{1}, \ldots, \mu_{N}$ are $N$ distinct complex numbers. Then,

$$
\mathbf{B}_{N}(\underline{\mu})^{-1}=\left(\mathbf{T}_{N-k}^{j}(\underline{\mu})\right)_{j, k=1}^{N} .
$$

From (3.18), we have

$$
z^{N_{2}} H(n)=2 \alpha_{0,+} \prod_{l=1}^{N}\left(z-\mu_{j}\right)=2 \alpha_{0,+} \sum_{l=0}^{N} \boldsymbol{\Psi}_{l}(\underline{\mu}) z^{N-l}
$$

Then $h_{l,+}=2 \alpha_{0,+} \boldsymbol{\Psi}_{l}(\underline{\mu}), 0 \leq l \leq N_{1}, h_{l,-}=2 \alpha_{0,+} \boldsymbol{\Psi}_{N-l}(\underline{\mu}), 0 \leq l \leq N_{2}-1$.

With these preparations, we can derive the following important result.

\section{Theorem 4.6 (Straightening out of the continuous flow).}

$$
\partial_{t_{\underline{m}}} \underline{\rho}\left(n, t_{\underline{m}}\right)=\underline{Y}^{(\underline{m})}, \quad m_{1} \geq 0, m_{2} \geq 1,
$$

where

$$
\underline{Y}^{(\underline{m})}=-2 \alpha \sum_{l=0}^{m_{1}} \gamma_{m_{1}-l} \underline{C}_{N-l}+2\left(\prod_{j=1}^{2 N+2} z_{j}\right)^{-\frac{1}{2}} \beta \sum_{l=0}^{m_{2}-1} \hat{\gamma}_{l} \underline{C}_{m_{2}-l}, \quad m_{1} \geq 0, m_{2} \geq 1,
$$

$\underline{\rho}\left(n, t_{m}\right)=\left(\rho_{1}\left(n, t_{m}\right), \ldots, \rho_{N}\left(n, t_{m}\right)\right), \underline{C}_{r}=\left(C_{1 r}, \ldots, C_{N r}\right), 1 \leq r \leq N$, and the recursive formula:

$$
\gamma_{0}=1, \quad \gamma_{1}=-\frac{\alpha_{1,+}}{\alpha_{0,+}}, \quad \gamma_{k}=\frac{1}{\alpha_{0,+}} \sum_{j=1}^{k}\left(-\alpha_{j,+}\right) \gamma_{k-j}
$$


D. Gong $\& 3$ X. Geng

$$
\hat{\gamma}_{0}=1, \quad \hat{\gamma}_{1}=-\frac{\alpha_{1,-}}{\alpha_{0-}}, \quad \hat{\gamma}_{k}=\frac{1}{\alpha_{0,-}} \sum_{j=1}^{k}\left(-\alpha_{j,-}\right) \hat{\gamma}_{k-j}
$$

Proof. From (3.9) and (3.10), we arrive at

$$
\begin{aligned}
& h_{k,+}(n)=\sum_{j=0}^{k} \alpha_{j,+} C_{k-j,+}^{(m)}(n), \\
& h_{k,-}(n)=\sum_{j=0}^{k} \alpha_{j,-} C_{k-j,-}^{(\underline{m})}(n),
\end{aligned}
$$

with $C_{k,+}^{(\underline{m})}(n)=2 S_{k-1}^{(2)}(n), C_{k,-}^{(\underline{m})}(n)=2 \hat{S}_{-k}^{(2)}(n)$, which implies by induction that

$$
\begin{aligned}
& C_{k,+}^{(\underline{m})}(n)=\frac{1}{\alpha_{0,+}} \sum_{l=0}^{k} \gamma_{l} h_{k-l,+}(n)=2 \sum_{l=0}^{k} \gamma_{l} \boldsymbol{\Psi}_{k-l}(\underline{\mu}), \\
& C_{k,-}^{(\underline{m})}(n)=\frac{1}{\alpha_{0,-}} \sum_{l=0}^{k} \hat{\gamma}_{l} h_{-k+l,-}(n)=\frac{2 \alpha_{0,+}}{\alpha_{0,-}} \sum_{l=0}^{k} \hat{\gamma}_{l} \Psi_{N-k+l}(\underline{\mu}) .
\end{aligned}
$$

In fact, it is obvious that $h_{0}(n)=\alpha_{0,+} C_{0}^{(m)}(n)=2 \alpha_{0,+}$. If (4.73) holds for a fixed $k \in \mathbb{N}_{0}$, then a direct calculation shows by (4.71) that

$$
\begin{aligned}
\alpha_{0,+} C_{k+1,+}^{(m)}= & h_{k+1,+}-\alpha_{1,+} C_{k}^{(m)}-\cdots-\alpha_{k,+} C_{1}^{(m)}-\alpha_{k+1,+} C_{0}^{(m)} \\
= & h_{k+1,+}+\left(-\alpha_{1,+} \gamma_{0}\right) h_{k}+\left(-\alpha_{1,+} \gamma_{1}-\alpha_{2,+} \gamma_{0}\right) h_{k-1,+} \\
& +\cdots+\left(-\alpha_{1,+} \gamma_{k}-\cdots-\alpha_{k+1,+}\right) h_{0,+} \\
& \sum_{l=0}^{k+1} \gamma_{l} h_{k+1-l,+} .
\end{aligned}
$$

Therefore (4.73) holds, in a similar way, we can prove (4.74).

From (3.24), (4.17), (4.73), (4.74), and use Theorems 4.3-4.5, we deduce that

$$
\begin{aligned}
\partial_{t_{\underline{m}}} \rho_{j}\left(n, t_{\underline{m}}\right)= & \sum_{l=1}^{N} \sum_{k=1}^{N} \frac{-C_{j l} \mu_{k}^{l-1}(n)}{\prod_{\substack{i=1 \\
i \neq k}}^{N}\left(\mu_{k}(n)-\mu_{i}(n)\right)} \\
& \times\left(\beta \sum_{m=0}^{m_{2}-1} C_{m,-}^{(\underline{m})}(n) \mu_{k}^{-m_{2}-m}+\alpha \sum_{m=0}^{m_{1}} C_{m,+}^{(\underline{m})}(n) \mu_{k}^{m_{1}-m}\right) \\
= & -2 \alpha \sum_{l=0}^{m_{1}} \gamma_{m_{1}-l} C_{j N-l}+2\left(\prod_{j=1}^{2 N+2} z_{j}\right)^{-\frac{1}{2}} \beta \sum_{l=0}^{m_{2}-1} \hat{\gamma}_{l} C_{j m_{2}-l} .
\end{aligned}
$$

This completes the proof of the theorem. 
In what follows, we shall discuss the straightening out of the discrete flow. Consider the fundamental solution matrix of (2.1) by

$$
M_{n}=\left(\hat{\chi}_{n}, \tilde{\chi}_{n}\right)=\left(\begin{array}{ll}
p^{(1)}(n) & p^{(2)}(n) \\
q^{(1)}(n) & q^{(2)}(n)
\end{array}\right), \quad M_{0}=\left(\begin{array}{ll}
1 & 0 \\
0 & 1
\end{array}\right),
$$

which can be expressed explicitly as

$$
M_{n+1}=U_{n} U_{n-1} \cdots U_{0} .
$$

By mathematical induction, we have

$$
\begin{aligned}
M_{1}= & \frac{1}{a(0)}\left(\begin{array}{cc}
0 & -a^{2}(0) \\
1 & \lambda-\lambda^{-1} b(0)
\end{array}\right), \\
M_{2}= & \frac{1}{a(0) a(1)}\left(\begin{array}{cc}
-a^{2}(1) & -\lambda a^{2}(1)+\lambda^{-1} a^{2}(1) b(0) \\
\lambda-\lambda^{-1} b(1) & \lambda^{2}-\left(a^{2}(0)+b(0)+b(1)\right)+\lambda^{-2} b(0) b(1)
\end{array}\right), \\
p^{(1)}(n)= & \frac{a^{2}(n-1)}{\prod_{j=0}^{n-1} a(j)} \\
& \left.\times\left\{\begin{array}{c}
-\lambda^{n-2}+\lambda^{n-4}\left(\sum_{j=1}^{n-3} a^{2}(j)+\sum_{j=1}^{n-2} b(j)\right.
\end{array}\right)-\cdots+(-1)^{n-1} \lambda^{-n+2} \prod_{j=1}^{n-2} b(j)\right\}, \\
p^{(2)}(n)= & \frac{a^{2}(n-1)}{\prod_{j=0}^{n-1} a(j)} \\
& \left.\times\left\{\begin{array}{l}
-\lambda^{n-1}+\lambda^{n-3}\left(\sum_{j=0}^{n-3} a^{2}(j)+\sum_{j=0}^{n-2} b(j)\right.
\end{array}\right)-\cdots+(-1)^{n} \lambda^{-n+1} \prod_{j=0}^{n-2} b(j)\right\},
\end{aligned}
$$

$$
\begin{aligned}
q^{(1)}(n)= & \frac{1}{\prod_{j=0}^{n-1} a(j)} \\
& \times\left\{\lambda^{n-1}-\lambda^{n-3}\left(\sum_{j=1}^{n-2} a^{2}(j)+\sum_{j=1}^{n-1} b(j)\right)+\cdots+(-1)^{n+1} \lambda^{-n+1} \prod_{j=1}^{n-1} b(j)\right\},
\end{aligned}
$$

$$
\begin{aligned}
q^{(2)}(n)= & \frac{1}{\prod_{j=0}^{n-1} a(j)} \\
& \times\left\{\lambda^{n}-\lambda^{n-2}\left(\sum_{j=0}^{n-2} a^{2}(j)+\sum_{j=0}^{n-1} b(j)\right)+\cdots+(-1)^{n} \lambda^{-n} \prod_{j=0}^{n-1} b(j)\right\} .
\end{aligned}
$$

The discrete commutative equation, $W_{n+1} U_{n}-U_{n} W_{n}=0$, is the key to straighten out of the discrete flow, which means that the solution space of the linear equation (2.1), 
$E \chi_{n}=U_{n} \chi_{n}$, is invariant under the action of $W_{n}$. Let $\alpha_{0,+} z^{-N_{2}} \varrho$ and $\chi_{n}$ be the eigenvalue and eigenfunction, respectively, of $W_{n}$ in the solution space. Then they satisfy

$$
E \chi_{n}=U_{n} \chi_{n}, \quad W_{n} \chi_{n}=\alpha_{0,+} z^{-N_{2}} \varrho \chi_{n} .
$$

It is easy to see that $\operatorname{det}\left(\alpha_{0,+} z^{-N_{2}} \varrho-W_{n}\right)=\alpha_{0,+}^{2} z^{-2 N_{2}} \varrho^{2}-G^{2}(n)-\lambda^{2} F(n) H(n)$, which yields to the algebraic curve $\mathcal{K}_{N}$. There are two eigenvalues $\varrho^{ \pm}= \pm \varrho$, and

$$
\varrho=\alpha_{0,+}^{-1} z^{N_{2}} \sqrt{G^{2}(n)+\lambda^{2} F(n) H(n)} .
$$

The eigenfunctions of the Lax matrix $W_{n}$ are called the Baker functions after some kind of normalization, which can be taken as

$$
\chi^{ \pm}(n)=c^{ \pm} \hat{\chi}_{n}+\tilde{\chi}_{n}
$$

with

$$
c^{ \pm}=\frac{G(0) \pm \alpha_{0,+} z^{-N_{2}} \varrho}{\lambda H(0)} .
$$

Proposition 4.7. Let $q^{ \pm}(n, \lambda)$ be the second component of the Baker function $\chi^{ \pm}(n, \lambda)$. Then

$$
q^{+}(n, \lambda) q^{-}(n, \lambda)=\frac{H(n)}{H(0)}=\prod_{j=1}^{N} \frac{z-\mu_{j}(n)}{z-\mu_{j}(0)} .
$$

Proof. Using (4.78) and the first expression of (3.2), we have

$$
W_{n} M_{n}=M_{n} W_{0},
$$

from which we can derive (4.87) through some direct calculations.

Using (4.84), (4.86) and (3.11), we obtain

$$
\begin{aligned}
\varrho & =\lambda^{2(N+1)}\left\{1+\alpha_{1}(\underline{z}) \lambda^{-2}+O\left(\lambda^{-4}\right)\right\}, \\
c^{+} & =-a^{2}(-1) \lambda^{-1}\left\{1+O\left(\lambda^{-2}\right)\right\}, \\
c^{-} & =-\lambda\left\{1+O\left(\lambda^{-2}\right)\right\} .
\end{aligned}
$$

Substituting (4.81) and (4.82) and the second expression of (4.89) into $q^{+}(n, \lambda)=c^{+} q^{(1)}(n)+q^{(2)}(n)$, we obtain

$$
q^{+}(n, \lambda)=\frac{\lambda^{n}}{\prod_{j=0}^{n-1} a(j)}\left\{1+O\left(\lambda^{-2}\right)\right\}
$$

for $\lambda \rightarrow \infty$. Using (3.87) and (3.90), we have

$$
q^{-}(n, \lambda)=\prod_{j=0}^{n-1} a(j) \lambda^{-n}\left\{1+O\left(\lambda^{-2}\right)\right\}
$$

for $\lambda \rightarrow \infty$. According to (4.86) and (4.89), it is easy to see that $\lambda c^{+}$and $\lambda c^{-}$are analytic functions of $z$, which can be viewed as the values of the single-valued function $[\lambda c](P)$ on 
the upper and lower sheets of $\mathcal{K}_{N}$, respectively. Moreover, it is obvious that the function $\lambda^{n-1} q^{(1)}(n)$ and $\lambda^{n} q^{(2)}(n)$ are $(n-1)$ th and $n$th order polynomials of $z=\lambda^{2}$, respectively. Therefore, the expressions

$$
\begin{aligned}
q^{ \pm}(2 k, \lambda) & =\left(\lambda c^{ \pm}\right)\left\{\frac{1}{\lambda} q^{(1)}(2 k)\right\}+q^{(2)}(2 k), \\
\lambda q^{ \pm}(2 k+1, \lambda) & =\left(\lambda c^{ \pm}\right) q^{(1)}(2 k+1)+\lambda q^{(2)}(2 k)
\end{aligned}
$$

determine two meromorphic functions of $z$ on $\mathcal{K}_{N}: q(2 k, P)$ and $[\lambda q](2 k+1, P)$. In the local coordinates $\zeta=z^{-1}, \hat{\xi}=z^{-N-1} y$, the equation of $\mathcal{K}_{N}$ near infinity is written as

$$
\hat{\xi}^{2}-R_{*}(\zeta)=0, \quad R_{*}(\zeta)=\prod_{j=1}^{2 N+2}\left(1-z_{j} \zeta\right)
$$

On $\mathcal{K}_{N}$ there are two infinities and two zeros

$$
P_{\infty \pm}=(\zeta=0, \hat{\xi}=\mp 1), \quad P_{0, \pm}=\left(z=0, y= \pm \sqrt{\prod_{j=1}^{2 N+2} z_{j}}\right)
$$

which are located on the upper $\left(P_{\infty_{-}}, P_{0,+}\right)$ and lower $\left(P_{\infty_{+}}, P_{0,-}\right)$ sheets, respectively. By (4.89) and (4.90), the principal asymptotic terms of the two meromorphic functions near $P_{\infty-}$ are

$$
q(2 k, P) \sim \frac{z^{k}}{\prod_{j=0}^{2 k-1} a(j)}, \quad[\lambda q](2 k+1, P) \sim \frac{z^{k+1}}{\prod_{j=0}^{2 k} a(j)}
$$

and their principal asymptotic terms nears $P_{\infty+}$ are

$$
q(2 k, P) \sim \prod_{j=0}^{2 k-1} a(j) z^{-k}, \quad[\lambda q](2 k+1, P) \sim \prod_{j=0}^{2 k} a(j) z^{-k} .
$$

Similarly, for $\lambda \rightarrow 0$ we obtain

$$
\begin{aligned}
& \varrho=\alpha_{0,-}+\alpha_{1,-} \lambda^{2}+O\left(\lambda^{4}\right), \quad c^{-}=\frac{a^{2}(-1)}{b(-1)} \lambda\left\{1+O\left(\lambda^{2}\right)\right\}, \\
& q^{-}(n, \lambda)=(-1)^{n} \prod_{j=0}^{n-1} \frac{b(j)}{a(j)} \lambda^{-n}\left\{1+O\left(\lambda^{2}\right)\right\}, \\
& q^{+}(n, \lambda)=(-1)^{n} \frac{b(0)}{b(n)} \prod_{j=0}^{n-1} \frac{a(j)}{b(j)} \lambda^{n}\left\{1+O\left(\lambda^{2}\right)\right\} .
\end{aligned}
$$

Therefore, the principal asymptotic terms nears $P_{0,+}$ are

$$
q(2 k, P) \sim \frac{b(0)}{b(2 k)} \prod_{j=0}^{2 k-1} \frac{a(j)}{b(j)} z^{k}, \quad[\lambda q](2 k+1, P) \sim-\frac{b(0)}{b(2 k+1)} \prod_{j=0}^{2 k} \frac{a(j)}{b(j)} z^{k+1}
$$


and their principal asymptotic terms near $P_{0,-}$ are

$$
q(2 k, P) \sim \prod_{j=0}^{2 k-1} \frac{a(j)}{b(j)} z^{-k}, \quad[\lambda q](2 k+1, P) \sim-\prod_{j=0}^{2 k} \frac{a(j)}{b(j)} z^{-k} .
$$

Based on the formula (4.87), and through an elementary analysis, it is easy to prove the following facts.

Proposition 4.8. The Baker function $q(2 k, P)$ has the following properties:

(1) $N$ simple poles at $\mu_{1}(0), \ldots, \mu_{N}(0)$ and two poles of $k$ th order at $P_{\infty-}$ and $P_{0,-}$;

(2) $N$ simple zeros at $\mu_{1}(2 k), \ldots, \mu_{N}(2 k)$ and two zeros of $k$ th order at $P_{\infty+}$ and $P_{0,+}$.

The Baker function $[\lambda q](2 k+1, P)$ is of the properties:

(1) $N$ simple poles at $\mu_{1}(0), \ldots, \mu_{N}(0)$ and a pole of $(k+1)$ th order at $P_{\infty-}$ and a pole of $k$ th order at $P_{0,-}$;

(2) $N$ simple zeros at $\mu_{1}(2 k+1), \ldots, \mu_{N}(2 k+1)$ and a zero of $k$ th order at $P_{\infty+}$ and a zero of $(k+1)$ th order at $P_{0,+}$.

\section{Theorem 4.9 (Straightening out of the discrete flow).}

$$
\begin{gathered}
\rho(2 k+1)-\rho(0)=2 k \Omega^{(0)}+\hbar_{+}, \quad(\bmod \mathcal{J}), \\
\rho(2 k)-\rho(0)=2 k \Omega^{(0)}, \quad(\bmod \mathcal{J})
\end{gathered}
$$

or

$$
\rho(n)-\rho(0)=\Omega^{(0)} n+\frac{1-(-1)^{n}}{4}\left(\hbar_{+}+\hbar_{-}\right), \quad(\bmod \mathcal{J})
$$

where

$$
\Omega^{(0)}=\frac{1}{2}\left(\hbar_{+}-\hbar_{-}\right), \quad \hbar_{ \pm}=\int_{P_{0, \pm}}^{P_{\infty \mp}} \omega
$$

Proof. For $n=2 k+1$, we introduce the meromorphic differential on $\mathcal{K}_{N}$ :

$$
\omega(2 k+1)=\left\{\frac{d}{d z} \ln [\lambda q](2 k+1, P)\right\} d z,
$$

which has poles at $\mu_{j}(0)$ and $\mu_{j}(2 k+1)$ with the residues -1 and 1 , respectively, and poles at $P_{\infty+}, P_{\infty--}, P_{0,-}, P_{0,+}$ with the residues $k,-(k+1),-k, k+1$, respectively. Let $\Omega$ be the Abel differential of the second kind, and $\omega(P, Q)$ be the normal Abel differential of the third kind with the residue $1,-1$ at $P, Q$, respectively, and the properties

$$
\int_{a_{j}} \omega(P, Q)=0, \quad \int_{b_{j}} \omega(P, Q)=2 \pi \sqrt{-1} \int_{Q}^{P} \omega_{j} .
$$

Here $\omega_{j}$ is the normalized Abel differential of the first kind given by (4.12). Then differential (4.103) can be written as a linear combination of $\Omega, \omega(P, Q)$ and $\omega_{j}$ in view of the poles, 
that is

$$
\begin{aligned}
\omega(2 k+1)= & \Omega+k \omega\left(P_{\infty+}, P_{\infty-}\right)+k \omega\left(P_{0,+}, P_{0,-}\right)+\omega\left(P_{0,+}, P_{\infty_{-}}\right) \\
& +\sum_{j=1}^{N} \omega\left(\mu_{j}(2 k+1), \mu_{j}(0)\right)+\sum_{j=1}^{N} e_{j} \omega_{j},
\end{aligned}
$$

where $e_{j}$ are some complex numbers. The integral of (4.105) along $a_{l}$ gives $e_{l}=2 \pi n_{l} \sqrt{-1}$, while the integral of (4.105) along $b_{l}$ yields

$$
\sum_{j=1}^{N} \int_{\mu_{j}(0)}^{\mu_{j}(2 k+1)} \omega_{l}=k\left(\int_{P_{0,+}}^{P_{\infty}-}-\int_{P_{0,-}}^{P_{\infty+}}\right) \omega_{l}+\int_{P_{0,+}}^{P_{\infty}-} \omega_{l}+m_{l}-\sum_{j=1}^{N} n_{j} \tau_{j l}
$$

where $n_{l}$ and $m_{l}$ are certain integers. Therefore, (4.99) holds. For $n=2 k$, we consider the meromorphic differential on $\mathcal{K}_{N}$

$$
\begin{aligned}
\omega(2 k) & =\left\{\frac{d}{d z} \ln q(2 k, P)\right\} d z \\
& =\Omega+k \omega\left(P_{\infty+}, P_{\infty-}\right)+k \omega\left(P_{0,+}, P_{0,-}\right)+\sum_{j=1}^{N} \omega\left(\mu_{j}(2 k), \mu_{j}(0)\right)+\sum_{j=1}^{N} \hat{e}_{j} \omega_{j} .
\end{aligned}
$$

Similar treatments lead to the proof of (4.100).

Now we have a clear evolution picture of the continuous flow and discrete flow through the Abel-Jacobi coordinates: (1) they are straightened out; (2) they commute each other. Therefore, the compatible solution of various flows are obtained simply by a linear superposition. Specifically, for the relativistic Toda hierarchy we have

$$
\rho\left(n, t_{\underline{m}}\right)=\Omega^{(0)} n+\underline{Y}^{(\underline{m})} t_{\underline{m}}+\frac{1-(-1)^{n}}{4}\left(\hbar_{+}+\hbar_{-}\right)+\rho_{0},
$$

where

$$
\rho_{0}=\sum_{k=1}^{N} \int_{P_{0}}^{P\left(\mu_{k}(0)\right)} \underline{\omega}
$$

\section{Quasi-Periodic Solutions}

Equation (4.108) gives the explicit solution of the relativistic Toda hierarchy in the Abel-Jacobi coordinate $\rho\left(n, t_{\underline{m}}\right)$. In order to get the explicit solutions of the relativistic Toda hierarchy in the original coordinates $a(n)$ and $b(n)$, we discuss the asymptotic properties of the fundamental meromorphic function $\phi\left(P, n, t_{\underline{m}}\right)$ on $\mathcal{K}_{N}$. Based on the divisor of $\phi\left(P, n, t_{\underline{m}}\right)$ and the properties of the normalized Abel differential of the third kind, we derive the explicit Riemann theta function representations of the meromorphic function $\phi\left(P, n, t_{\underline{m}}\right)$, from which explicit Riemann theta function representations of solutions for the relativistic Toda hierarchy are obtained in the original coordinates. The class of solutions given by the Riemann theta function is quasi-periodic because the Riemann theta function 
is multivariable and periodic for each variable, but usually it is not a periodic function for all the variables.

Let us consider the hyperelliptic curve $\mathcal{K}_{N}: y^{2}-\alpha_{0,+}^{-2} z^{2 N_{2}} R(z)=0$ and the fundamental meromorphic function $\phi\left(P, n, t_{\underline{m}}\right)$ on $\mathcal{K}_{N}$

$$
\phi\left(P, n, \underline{t}_{\underline{m}}\right)=\frac{\alpha_{0,+} z^{-N_{2}} y-G\left(n, t_{\underline{m}}\right)}{F\left(n, t_{\underline{m}}\right)}=\frac{z H\left(n, t_{\underline{m}}\right)}{\alpha_{0,+} z^{-N_{2}} y+G\left(n, t_{\underline{m}}\right)},
$$

where $P=(z, y) \in \mathcal{K}_{N} \backslash\left\{P_{\infty+}, P_{\infty-}, P_{0,+}, P_{0,-}\right\}$. The divisor of $\phi\left(\cdot, n, t_{\underline{m}}\right)$ gives by

$$
\left(\phi\left(\cdot, n, t_{\underline{m}}\right)\right)=D_{\underline{\hat{\mu}}\left(n, t_{\underline{m}}\right)}-D_{\underline{\hat{\mu}}^{-}\left(n, t_{\underline{m}}\right)}+P_{0,+}-P_{\infty-}
$$

with

$$
\underline{\hat{\mu}}\left(n, t_{\underline{m}}\right)=\left\{\hat{\mu}_{1}\left(n, t_{\underline{m}}\right), \ldots, \hat{\mu}_{N}\left(n, t_{\underline{m}}\right)\right\}, \quad \underline{\hat{\mu}}^{-}\left(n, t_{\underline{m}}\right)=\left\{\hat{\mu}_{1}^{-}\left(n, t_{\underline{m}}\right), \ldots, \hat{\mu}_{N}^{-}\left(n, t_{\underline{m}}\right)\right\} .
$$

Next we turn to asymptotic properties of $\phi$ in a neighborhood of $P_{\infty \pm}$ and $P_{0, \pm}$.

Lemma 5.1. Suppose that $a\left(n, t_{\underline{m}}\right)$ and $b\left(n, t_{\underline{m}}\right)$ satisfy the hierarchy of relativistic Toda equations (2.9). Let $z_{j} \in \mathbb{C} \backslash\{0\},(1 \leq j \leq 2 \bar{N}+2)$, and $P=(z, y) \in \mathcal{K}_{N} \backslash\left\{P_{\infty_{+}}, P_{\infty_{-}}\right.$, $\left.P_{0,+}, P_{0,-}\right\}$ and $\left(n, t_{\underline{m}}\right) \in \mathbb{Z} \times \mathbb{R}$. Then

$$
\begin{aligned}
& \phi(P) \underset{\zeta \rightarrow 0}{=}\left\{\begin{array}{ll}
-1+O(\zeta), & \text { as } P \rightarrow P_{\infty+}, \\
-\frac{1}{\left(a^{-}\right)^{2}} \zeta^{-1}+O(1), & \text { as } P \rightarrow P_{\infty-} ;
\end{array} \quad \zeta=\frac{1}{z},\right. \\
& \phi(P) \underset{\zeta \rightarrow 0}{=} \begin{cases}\frac{1}{b} \zeta+O\left(\zeta^{2}\right), & \text { as } P \rightarrow P_{0,+}, \\
\frac{b^{-}}{\left(a^{-}\right)^{2}}+O(\zeta), & \text { as } P \rightarrow P_{0,-} .\end{cases}
\end{aligned}
$$

Proof. Introducing the local coordinate $\zeta=z^{-1}$ near $P_{\infty \pm}$ and $\zeta=z$ near $P_{0, \pm}$, from (3.5), (3.11) and (4.9), we have

$$
\begin{aligned}
& G \underset{\zeta \rightarrow 0}{=} \begin{cases}\zeta^{-N_{1}-1}\left(g_{0,+}+g_{1,+} \zeta+g_{2,+} \zeta^{2}+g_{3,+} \zeta^{3}+O\left(\zeta^{4}\right)\right), & \text { as } P \rightarrow P_{\infty \pm}, \\
\zeta^{-N_{2}}\left(g_{0,-}+O(\zeta)\right), & \text { as } P \rightarrow P_{0, \pm},\end{cases} \\
& F^{-1}=-\frac{z^{N_{2}}}{2 \alpha_{0,+}\left(a^{-}\right)^{2}} \prod_{j=1}^{N}\left(z-\mu_{j}^{-}\right)^{-1} \text { as } P \rightarrow P_{\infty \pm}, \\
&= \begin{cases}-\frac{\zeta^{N_{1}}}{2 \alpha_{0,+}\left(a^{-}\right)^{2}} \prod_{j=1}^{N}\left(1-\mu_{j}^{-} \zeta\right)^{-1}, & \\
\frac{(-1)^{N+1} z^{N_{1}}}{2 \alpha_{0,+}\left(a^{-}\right)^{2} \prod_{j=1}^{N} \mu_{j}^{-}} \prod_{j=1}^{N}\left(1-\left(\mu_{j}^{-}\right)^{-1}\right)^{-1}, & \text { as } P \rightarrow P_{0, \pm},\end{cases}
\end{aligned}
$$




$$
\underset{\zeta \rightarrow 0}{=} \begin{cases}-\frac{\zeta^{N_{1}}}{2 \alpha_{0,+}\left(a^{-}\right)^{2}}\left(1+\sum_{j=1}^{N} \mu_{j}^{-} \zeta+O\left(\zeta^{2}\right)\right), & \text { as } P \rightarrow P_{\infty \pm}, \\ -\frac{\zeta^{N_{1}} b^{-}}{2 \alpha_{0,-}\left(a^{-}\right)^{2}}\left(1+\sum_{j=1}^{N}\left(\mu_{j}^{-}\right)^{-1} \zeta+O\left(\zeta^{2}\right)\right), & \text { as } P \rightarrow P_{0, \pm},\end{cases}
$$

$$
y \underset{\zeta \rightarrow 0}{=} \begin{cases}\mp \zeta^{-N-1} \prod_{j=1}^{2 N+2}\left(1-z_{j} \zeta\right)^{\frac{1}{2}} \underset{\zeta \rightarrow 0}{=} \mp \zeta^{-N-1} \sum_{k=0}^{\infty} \alpha_{k}(\underline{z}) \zeta^{k}, \quad \text { as } P \rightarrow P_{\infty \pm}, \\ \left(\prod_{j=1}^{2 N+2} z_{j}\right)^{\frac{1}{2}} \prod_{j=1}^{2 N+2}\left(1-z_{j}^{-1} \zeta\right)^{\frac{1}{2}} \underset{\zeta \rightarrow 0}{=} \pm \frac{\alpha_{0,-}}{\alpha_{0,+}} \sum_{k=0}^{\infty} \alpha_{k}\left(\underline{z}^{-1}\right) \zeta^{k}, & \text { as } P \rightarrow P_{0, \pm},\end{cases}
$$

where $\underline{z}=\left(z_{1}, \ldots, z_{2 N+2}\right), \underline{z}^{-1}=\left(z_{1}^{-1}, \ldots, z_{2 N+2}^{-1}\right)$,

$$
\begin{aligned}
& \alpha_{0}\left(\underline{z}^{ \pm 1}\right)=1, \\
& \alpha_{k}\left(\underline{z}^{ \pm 1}\right)=\sum_{\substack{j_{1}, \ldots, j_{2 N+2}=0 \\
j_{1}+\cdots+j_{2 N+2}=k}}^{k} \frac{\left(2 j_{1}\right) ! \cdots\left(2 j_{2 N+2}\right) ! z_{1}^{ \pm j_{1}} \cdots z_{2 N+2}^{ \pm j_{2 N+2}}}{2^{2 k}\left(j_{1} !\right)^{2} \cdots\left(j_{2 N+2} !\right)^{2}\left(2 j_{1}-1\right) \cdots\left(2 j_{2 N+2}-1\right)}, \quad k \in \mathbb{N} .
\end{aligned}
$$

Then according to the definition of $\phi$ in (4.9), we finally obtain that

$$
\begin{aligned}
& \phi\left(P, n, t_{m}\right)=\left(\alpha_{0,+} z^{-N_{1}} y-G\right) \times F^{-1} \\
& \underset{\zeta \rightarrow 0}{=} \begin{cases}{\left[\mp \alpha_{0,+}\left(1+\alpha_{1}(\underline{\lambda}) \zeta+\alpha_{2}(\underline{\lambda}) \zeta^{2}+\alpha_{3}(\underline{\lambda}) \zeta^{3}+O\left(\zeta^{4}\right)\right)\right.} & \\
\left.-\left(g_{0,+}+g_{1,+} \zeta+g_{2,+} \zeta^{2}+g_{3,+} \zeta^{3}+O\left(\zeta^{4}\right)\right)\right] & \\
\times\left(1+\sum_{j=1}^{N} \mu_{j}^{-} \zeta+O\left(\zeta^{2}\right)\right) \frac{-1}{2 \alpha_{0,+}\left(a^{-}\right)^{2}} \zeta^{-1}, & \text { as } P \rightarrow P_{\infty \pm}, \\
-\left[ \pm \alpha_{0,-}(1+O(\zeta))-\left(g_{0,-}+O(\zeta)\right)\right] \frac{b^{-}}{2 \alpha_{0,-}\left(a^{-}\right)^{2}}(1+O(\zeta)), & \text { as } P \rightarrow P_{0, \pm},\end{cases} \\
& \underset{\zeta \rightarrow 0}{=} \begin{cases}-1+O(\zeta) & \text { as } P \rightarrow P_{\infty+}, \\
-\frac{1}{\left(a^{-}\right)^{2}} \zeta^{-1}+O(1), & \text { as } P \rightarrow P_{\infty-}, \\
\frac{1}{b} \zeta+O\left(\zeta^{2}\right), & \text { as } P \rightarrow P_{0,+}, \\
\frac{b^{-}}{\left(a^{-}\right)^{2}}+O(\zeta), & \text { as } P \rightarrow P_{0,-},\end{cases}
\end{aligned}
$$

which proves (5.1) and (5.2). 
Next, we shall derive the representation of $\phi, a^{2}(n)$ and $b(n)$ in terms of the Riemann theta functions. Let $\omega_{P_{0,+}, P_{\infty-}}^{(3)}$ be the normal differential of the third kind holomorphic on $\mathcal{K}_{N} \backslash\left\{P_{0,+}, P_{\infty-}\right\}$ with simple poles at $P_{0,+}$ and $P_{\infty-}$, corresponding residues 1 and -1 , respectively, which can be expressed as

$$
\omega_{P_{0,+}, P_{\infty-}}^{(3)}=\frac{y+y_{0,-}}{2 z} \frac{d z}{y}+\frac{1}{2 y} \prod_{j=1}^{N}\left(z-\beta_{j}\right) d z, \quad P_{0,-}=\left(0, y_{0,-}\right)
$$

where $\beta_{j} \in \mathbb{C}, j=1, \ldots, N$, are constants that are determined by

$$
\int_{a_{j}} \omega_{P_{0,+}, P_{\infty-}}^{(3)}=0, \quad j=1, \ldots, N
$$

If the local coordinate near $P_{\infty \pm}$ is given by $\zeta=z^{-1}$, then we have the asymptotic expansions of $\omega_{P_{0,+}, P_{\infty-}}^{(3)}$ near $P_{\infty \pm}$ :

$$
\omega_{P_{0,+}, P_{\infty-}}^{(3)}(P) \underset{\zeta \rightarrow 0}{=}\left\{\begin{array}{c}
0 \\
-\zeta^{-1}
\end{array}\right\} d \zeta \pm\left[\sum_{q=0}^{\infty}(q+1) \omega_{q+1}^{\infty} \zeta^{q}\right] d \zeta, \quad \text { as } P \rightarrow P_{\infty, \pm}
$$

and similarly (using the local coordinate $\zeta=\lambda$ near $P_{0, \pm}$ )

$$
\omega_{P_{0,+}, P_{\infty-}}^{(3)}(P) \underset{\zeta \rightarrow 0}{=}\left\{\begin{array}{l}
\zeta^{-1} \\
0
\end{array}\right\} d \zeta \pm\left[\sum_{q=0}^{\infty}(q+1) \omega_{q+1}^{0} \zeta^{q}\right] d \zeta, \quad \text { as } P \rightarrow P_{0, \pm}
$$

In particular,

$$
\begin{aligned}
& \int_{P_{0}}^{P} \omega_{P_{0,+}, P_{\infty}-}^{(3)}=\left\{\begin{array}{c}
0 \\
-\ln (\zeta)
\end{array}\right\}+\omega_{0}^{\infty \pm} \pm \omega_{1}^{\infty} \zeta+O\left(\zeta^{2}\right), \quad \text { as } P \rightarrow P_{\infty, \pm}, \\
& \int_{P_{0}}^{P} \omega_{P_{0,+}, P_{\infty}-}^{(3)}=\left\{\begin{array}{c}
\ln (\zeta) \\
0
\end{array}\right\}+\omega_{0}^{0 \pm} \pm \omega_{1}^{0} \zeta+O\left(\zeta^{2}\right), \quad \text { as } P \rightarrow P_{0, \pm} .
\end{aligned}
$$

Given these preparations, the theta function representations for $\phi\left(P, n, t_{\underline{m}}\right), a^{2}\left(n, t_{\underline{m}}\right)$, $b\left(n, t_{\underline{m}}\right)$ then read as follows.

Theorem 5.2. Let $P=(z, y) \in \mathcal{K}_{N} \backslash\left\{P_{\infty+}, P_{\infty_{-}}, P_{0,+}, P_{0,-}\right\},\left(n, t_{\underline{m}}\right) \in \mathbb{Z} \times \mathbb{R}$, Suppose $a\left(n, t_{\underline{m}}\right), b\left(n, t_{\underline{m}}\right)$ satisfy the hierarchy of relativistic Toda equations (2.9), and assume that $z_{j}, 1 \leq j \leq 2 N+2$, in (4.2) satisfy $z_{j} \in \mathbb{C} \backslash\{0\}$ and $z_{j} \neq z_{k}$ as $j \neq k$. Moreover, suppose that $D_{\underline{\hat{\mu}}\left(n, t_{\underline{m}}\right)}$ is nonspecial for each $\left(n, t_{\underline{m}}\right) \in \mathbb{Z} \times \mathbb{R}$. Then $\phi$ admits the following representation

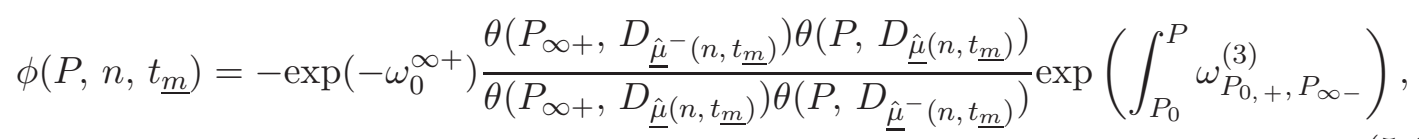


Finally, $a^{2}\left(n, t_{\underline{m}}\right), b\left(n, t_{\underline{m}}\right)$ are of the form

$$
\begin{aligned}
a^{2}\left(n, t_{\underline{m}}\right) & =\exp \left(\omega_{0}^{\infty+}-\omega_{0}^{\infty-}\right) \frac{\theta\left(P_{\infty+}, D_{\underline{\hat{\mu}}^{+}\left(n, t_{\underline{m}}\right)}\right) \theta\left(P_{\infty-}, D_{\underline{\hat{\mu}}\left(n, t_{\underline{m}}\right)}\right)}{\theta\left(P_{\infty+}, D_{\underline{\hat{\mu}}\left(n, t_{\underline{m}}\right)}\right) \theta\left(P_{\infty-}, D_{\underline{\hat{\mu}}^{+}\left(n, t_{\underline{m}}\right)}\right)}, \\
b\left(n, t_{\underline{m}}\right) & =-\exp \left(\omega_{0}^{\infty+}-\omega_{0}^{0+}\right) \frac{\theta\left(P_{\infty+}, D_{\underline{\hat{\mu}}\left(n, t_{\underline{m}}\right)}\right) \theta\left(P_{0,+}, D_{\underline{\hat{\mu}}^{-}\left(n, t_{\underline{m}}\right)}\right)}{\theta\left(P_{\infty+}, D_{\underline{\hat{\mu}}^{-}\left(n, t_{\underline{m}}\right)}\right) \theta\left(P_{0,+}, D_{\underline{\hat{\mu}}\left(n, t_{\underline{m}}\right)}\right)} .
\end{aligned}
$$

Proof. To prove (5.12), we denote its right-hand side by $\tilde{\phi}$. From (4.6), one can conclude that it has simple zeros at $P_{0,+}$ and $\hat{\mu}_{k}\left(n, t_{\underline{m}}\right), k=1, \ldots, N$, and simple poles at $P_{\infty-}$ and $\hat{\mu}_{k}^{-}\left(n, t_{\underline{m}}\right), k=1, \ldots, N$. On the other hand, by (5.10), (5.11) and the expression of $\tilde{\phi}$, we know that $\tilde{\phi}$ shares the same properties with $\phi$. Then, according to the Riemann-Roch theorem, $\frac{\tilde{\phi}}{\phi}=c$ for some constant $c \in \mathbb{C}$. Using (5.10) and the first expression of (5.1), we can infer that

$$
\frac{\tilde{\phi}}{\phi} \underset{\zeta \rightarrow 0}{=} \frac{(-1+O(\zeta))(1+O(\zeta))}{-1+O(\zeta)} \underset{\zeta \rightarrow 0}{=} 1+O(\zeta),
$$

hence $c=1$. This proves (5.12).

Next, we turn to the proof of (5.13). First, if the local coordinate $\zeta=\frac{1}{z}$ is introduced near $P_{\infty \pm}$, we can conclude from the definition (4.12) of the normalized basis $\omega_{j}$ that

$$
\begin{aligned}
\underline{\omega} & =\left(\omega_{1}, \ldots, \omega_{N}\right)=\mp \sum_{l=1}^{N} \frac{\underline{C}_{l} z^{l-1} d z}{\prod_{j=1}^{2 N+2}\left(z-z_{j}\right)^{\frac{1}{2}}} \\
& = \pm \sum_{l=1}^{N} \frac{\underline{C}_{l} \zeta^{N-j} d \zeta}{\prod_{j=1}^{2 N+2}\left(1-z_{j} \zeta\right)^{\frac{1}{2}}} \underset{\zeta \rightarrow 0}{=} \pm\left(\underline{C}_{N}+O(\zeta)\right) d \zeta, \quad \text { as } P \rightarrow P_{\infty \pm} .
\end{aligned}
$$

Hence, the Abel map

$$
\begin{aligned}
\mathcal{A}(P) & =\int_{P_{0}}^{P} \underline{\omega}=\int_{P_{0}}^{P_{\infty \pm}} \underline{\omega}+\int_{P_{\infty \pm}}^{P} \underline{\omega} \\
& =\mathcal{A}\left(P_{\infty \pm}\right)+\int_{P_{\infty \pm}}^{P} \underline{\omega} \\
& =\mathcal{A}\left(P_{\infty \pm}\right) \pm \underline{C}_{N} \zeta+O\left(\zeta^{2}\right) .
\end{aligned}
$$

Therefore, expanding the ratios of Riemann theta functions in (5.12), we obtain

$$
\begin{aligned}
& \frac{\theta\left(P, D_{\underline{\hat{\mu}}\left(n, t_{\underline{m}}\right)}\right)}{\theta\left(P_{\infty-}, D_{\underline{\hat{\mu}}\left(n, t_{\underline{m}}\right)}\right)} \underset{\zeta \rightarrow 0}{=} \frac{\theta\left(\ldots, \Lambda_{j}-\mathcal{A}\left(P_{\infty-}\right)+\underline{C}_{N} \zeta+O\left(\zeta^{2}\right)+\rho_{j}, \ldots\right)}{\theta\left(P_{\infty-}, D_{\underline{\hat{\mu}}\left(n, t_{\underline{m}}\right)}\right)} \\
& \underset{\zeta \rightarrow 0}{=} 1+\frac{\left.\sum_{j=1}^{N} C_{j N} \partial_{\omega_{j}} \theta\left(\Lambda_{j}-\mathcal{A}\left(P_{\infty-}\right)+\underline{\omega}+\underline{\rho}\right)\right|_{\underline{\omega}=0}}{\theta\left(P_{\infty-}, D_{\underline{\hat{\mu}}\left(n, t_{\underline{m}}\right)}\right)} \zeta+O\left(\zeta^{2}\right) \\
& \underset{\zeta \rightarrow 0}{=} 1+\sum_{j=1}^{N} C_{j N} \partial_{\omega_{j}} \ln \theta\left(P_{\infty-}, D_{\underline{\hat{\mu}}\left(n, t_{\underline{m}}\right)}\right) \zeta+O\left(\zeta^{2}\right) \quad \text { as } P \rightarrow P_{\infty_{-}-},
\end{aligned}
$$


D. Gong $\mathcal{E}$ X. Geng

and the same formula for the theta function ratio involving $D_{\hat{\mu}^{-}\left(n, t_{m}\right)}$, that is

$$
\begin{aligned}
& \frac{\theta\left(P, D_{\underline{\hat{\mu}}^{-}\left(n, t_{\underline{m}}\right)}\right)}{\theta\left(P_{\infty-}, D_{\underline{\hat{\mu}}^{-}\left(n, t_{\underline{m}}\right)}\right)} \underset{\zeta \rightarrow 0}{=} 1+\sum_{j=1}^{N} C_{j N} \partial_{\omega_{j}} \ln \theta\left(P_{\infty-}, D_{\underline{\hat{\mu}}^{-}\left(n, t_{\underline{m}}\right)}\right) \zeta \\
& +O\left(\zeta^{2}\right) \quad \text { as } P \rightarrow P_{\infty-} .
\end{aligned}
$$

Finally, from the representation (5.12) of $\phi$, we get

$$
\begin{aligned}
\phi \underset{\zeta \rightarrow 0}{=} & -\exp \left(\omega_{0}^{\infty-}-\omega_{0}^{\infty+}\right) \frac{\theta\left(P_{\infty+}, D_{\underline{\hat{\mu}}^{-}\left(n, t_{\underline{m}}\right)}\right) \theta\left(P_{\infty-}, D_{\underline{\hat{\mu}}\left(n, t_{\underline{m}}\right)}\right)}{\theta\left(P_{\infty+}, D_{\underline{\hat{\mu}}\left(n, t_{\underline{m}}\right)}\right) \theta\left(P_{\infty-}, D_{\underline{\hat{\mu}}^{-}\left(n, t_{\underline{m}}\right)}\right)} \zeta^{-1} \\
& +O(1) \quad \text { as } P \rightarrow P_{\infty-},
\end{aligned}
$$

which together with the second expression of (5.1), shows that

$$
a^{2}\left(n-1, t_{\underline{m}}\right)=\exp \left(\omega_{0}^{\infty+}-\omega_{0}^{\infty-}\right) \frac{\theta\left(P_{\infty+}, D_{\underline{\hat{\mu}}\left(n, t_{\underline{m}}\right)}\right) \theta\left(P_{\infty-}, D_{\underline{\underline{\mu}}^{-}\left(n, t_{\underline{m}}\right)}\right)}{\theta\left(P_{\infty+}, D_{\underline{\hat{\mu}}^{-}\left(n, t_{\underline{m}}\right)}\right) \theta\left(P_{\infty-}, D_{\underline{\hat{\mu}}\left(n, t_{\underline{m}}\right)}\right)} .
$$

Therefore,

$$
a^{2}\left(n, t_{\underline{m}}\right)=\exp \left(\omega_{0}^{\infty+}-\omega_{0}^{\infty-}\right) \frac{\theta\left(P_{\infty+}, D_{\underline{\hat{\mu}}^{+}\left(n, t_{\underline{m}}\right)}\right) \theta\left(P_{\infty-}, D_{\underline{\hat{\mu}}\left(n, t_{\underline{m}}\right)}\right)}{\theta\left(P_{\infty+}, D_{\underline{\hat{\mu}}\left(n, t_{\underline{m}}\right)}\right) \theta\left(P_{\infty-}, D_{\underline{\hat{\mu}}^{+}\left(n, t_{\underline{m}}\right)}\right)},
$$

which proves $(5.13)$. If we introduce the local coordinate $\zeta=z$ near $P_{0,+}$, in the similar way, from the representation $(5.16)$ of $\phi$, we get

$$
\begin{aligned}
\phi \underset{\zeta \rightarrow 0}{=} & -\exp \left(\omega_{0}^{0+}-\omega_{0}^{\infty+}\right) \frac{\theta\left(P_{\infty+}, D_{\underline{\hat{\mu}}^{-}\left(n, t_{\underline{m}}\right.}\right) \theta\left(P_{0,-}, D_{\underline{\hat{\mu}}\left(n, t_{\underline{m}}\right)}\right)}{\theta\left(P_{\infty+}, D_{\underline{\hat{\mu}}\left(n, t_{\underline{m}}\right)}\right) \theta\left(P_{0,+}, D_{\underline{\hat{\mu}}^{-}\left(n, t_{\underline{m}}\right)}\right)} \zeta \\
& +O\left(\zeta^{2}\right) \quad \text { as } P \rightarrow P_{0,+},
\end{aligned}
$$

together with the first expression of (5.2), this shows that

$$
b\left(n, t_{\underline{m}}\right)=-\exp \left(\omega_{0}^{\infty+}-\omega_{0}^{0,+}\right) \frac{\theta\left(P_{\infty+}, D_{\underline{\hat{\mu}}\left(n, t_{\underline{m}}\right)}\right) \theta\left(P_{0,+}, D_{\underline{\hat{\mu}}^{-}\left(n, t_{\underline{m}}\right)}\right)}{\theta\left(P_{\infty+}, D_{\underline{\hat{\mu}}^{-}\left(n, t_{\underline{m}}\right)}\right) \theta\left(P_{0,+}, D_{\underline{\hat{\mu}}\left(n, t_{\underline{m}}\right)}\right)},
$$

which proves (5.14).

\section{Acknowledgments}

This work was supported by National Natural Science Foundation of China (project no. 11171312).

\section{References}

[1] S. J. Alber, On finite-zone solutions of relativistic Toda lattices, Lett. Math. Phys. 17 (1989) 149-155. 
[2] M. Bruschi and O. Ragnisco, Recursion operator and Bäcklund transformations for the Ruijsenaars-Toda lattice, Phys. Lett. A 129 (1988) 21-25.

[3] M. Bruschi and O. Ragnisco, Lax representation and complete integrability for the periodic relativistic Toda lattice, Phys. Lett. A 134 (1989) 365-370.

[4] M. Bruschi and O. Ragnisco, The periodic relativistic Toda lattice: Direct and inverse problem, Inverse Problems 5 (1989) 389-405.

[5] W. Bulla, F. Gesztesy, H. Holden and G. Teschl, Algebro-geometric quasi-periodic finite-gap solutions of the Toda and Kac-van Moerbeke hierarchies, Mem. Amer. Math. Soc. 135(641) (1998) $1-79$.

[6] C. W. Cao, X. G. Geng and H. Y. Wang, Algebro-geometric solution of the $2+1$ dimensional Burgers equation with a discrete variable, J. Math. Phys. 43 (2002) 621-643.

[7] H. H. Dai and X. G. Geng, Explicit solutions of the 2+1-dimensional modified Toda lattice through straightening out of the relativistic Toda flows, J. Phys. Soc. Japan 72 (2003) 30633069.

[8] X. G. Geng, H. H. Dai and C. W. Cao, Algebro-geometric constructions of the discrete Ablowitz-Ladik flows and applications, J. Math. Phys. 44 (2003) 4573-4588.

[9] X. G. Geng, H. H. Dai and J. Y. Zhu, Decomposition of the discrete Ablowitz-Ladik hierarchy, Stud. Appl. Math. 118 (2007) 281-312.

[10] J. S. Geronimo, F. Gesztesy and H. Holden, Algebro-geometric solutions of the Baxter-Szegö difference equation, Comm. Math. Phys. 258 (2005) 149-177.

[11] P. Griffiths and J. Harris, Principles of Algebraic Geometry (Wiley, New York, 1994).

[12] E. G. Kalnins and W. Miller Jr., Jacobi elliptic coordinates, functions of Heun and Lamé type and the Niven transform, Regul. Chaotic Dyn. 10 (2005) 487-508.

[13] D. Mumford, Tata Lectures on Theta II (Birkhäuser, Boston, 1984).

[14] W. Oevel, B. Fuchssteiner and H. W. Zhang, Master symmetries, angle variables and recursion operator of the relativistic Toda lattice, J. Math. Phys. 30 (1989) 2664-2676.

[15] Y. Ohta, K. Kajiwara, J. Matsukidaira and J. Satsuma, Casorati determinant solution for the relativistic Toda lattice equation, J. Math. Phys. 34 (1993) 5190-5204.

[16] S. N. M. Ruijsenaars, Relativistic Toda systems, Comm. Math. Phys. 133 (1990) 217-247.

[17] Y. B. Suris, Algebraic structure of discrete-time and relativistic Toda lattices, Phys. Lett. A 156 (1991) 467-474.

[18] Y. T. Wu and X. G. Geng, A discrete version of classical integrable system of Neumann type related to relativistic Toda lattice, in Nonlinear Partial Differential Equations and Applications (World Scientific Publishing, River Edge, NJ, 1998), pp. 186-192.

[19] R. G. Zhou and Q. Y. Jiang, A Darboux transformation and an exact solution for the relativistic Toda lattice equation, J. Phys. A: Math. Gen. 38 (2005) 7735-7742. 